\title{
Nonlinear Characteristics of Three-parameter Fluid Viscous Damper with Considering of Principal Stiffness and Hydraulic Stiffness
}

Xiaolei Jiao ( $\nabla_{\text {jiaoxlei@mail.sysu.edu.cn ) }}$

Sun Yat-Sen University https://orcid.org/0000-0001-8072-0283

Jinxiu Zhang

Sun Yat-Sen University

Wenbo Li

Beijing Institute of Control Engineering

Jingjing Mu

China Aerospace Science and Technology Corp

\section{Research Article}

Keywords: Nonlinear characteristics, Three-parameter fluid viscous damper , principal stiffness, hydraulic stiffness

Posted Date: July 26th, 2021

DOl: https://doi.org/10.21203/rs.3.rs-721905/v1

License: (9) This work is licensed under a Creative Commons Attribution 4.0 International License. Read Full License 


\title{
Nonlinear characteristics of three-parameter fluid viscous damper with considering of principal stiffness and hydraulic stiffness
}

\author{
Xiaolei Jiao ${ }^{1}$, Jinxiu Zhang ${ }^{1}$, Wenbo $\mathrm{Li}^{2}$, Jingjing $\mathrm{Mu}^{3}$ \\ 1. School of Aeronautics and Astronautics, Sun Yat-Sen University, Shenzhen 518107, \\ China \\ 2. Beijing Institute of Control Engineering, Beijing 100190, China \\ 3. China Aerospace Science and Technology Corporation, Beijing 100048, China
}

Wenbo Li (Corresponding Author)

E-mail: liwenbo_502@163.com

Beijing Institute of Control Engineering, Beijing 100190, China

\begin{abstract}
The three-parameter fluid viscous damper is used to isolate micro-vibration produced by control torque gyro (CMG) in satellite. In this paper, the damper is simplified by a single tube fluid viscous damper and two springs connected to the damping piston. With consideration of the principal stiffness of the bellows and the contraction and expansion effect of the damping orifice, the approximate analytical nonlinear model of the damper is established and verified by the computation fluid mechanics (CFD) method. Based on this analytical model, the displacement response of the damper and correction coefficient of hydraulic resistance are analyzed, the nonlinear characteristics in the frequency domain are also revealed. Furthermore, the energy consumptions of the nonlinear model and linear model are researched. The results show that the damper has an obvious amplitude at the first resonance peak, but not obvious at the second resonance peak. The vibration amplitude of the damping piston is only um level in the highfrequency domain. The correction coefficient of the hydraulic resistance at the resonance peak is much higher than other frequencies, which causes a significant nonlinear behavior. In addition, the energy
\end{abstract}


consumption of the nonlinear model is larger than that of the linear model at the resonance peak, and the larger the resonance peak, the more obvious the phenomenon is. This means that the nonlinear damping can be used to further improve the suppression of the resonance peak of the three-parameter fluid viscous damper.

Keywords: Nonlinear characteristics $\cdot$ Three-parameter fluid viscous

damper $\cdot$ principal stiffness $\cdot$ hydraulic stiffness

\section{Introduction}

The control torque gyro and reaction flywheel are the actuators on the spacecraft, whose output moment is used to control the attitude of the spacecraft. Due to the static and dynamic unbalance of the rotor, micro-vibration is easy to occur at high speed. Micro-vibration has characteristics of small amplitude and high frequency [1]. If not suppressed, it will cause a serious influence on the high-precision payload on spacecraft. The research shows that the installation of a vibration damper on the propagation path of micro-vibration is an effective means to suppress micro-vibration [1].

At present, micro-vibration isolation devices are divided into passive vibration isolation devices, active vibration isolation devices, hybrid vibration isolation devices, and semi-active vibration isolation devices [1]. Among the passive vibration isolation devices, one kind of vibration damper is widely used, namely a three-parameter fluid viscous damper. This kind of vibration damper was first proposed in the 1990s for the vibration isolation of reaction flywheel on the Hubble Space Telescope, and the vibration isolation effect is perfect after on-orbit verification [2]. Later, it was widely used to isolate the vibration of the control moment gyroscope and reaction flywheel on conventional satellites. When a three-parameter fluid viscous damper is used to suppress micro-vibration, it is usually assembled as a vibration isolation platform to achieve six degrees of freedom vibration isolation. For example, the Fengyun-4 satellite 
uses three three-parameter fluid viscous dampers to constitute a vibration isolation platform to suppress the micro-vibration generated by the reaction flywheel. Through testing, the attenuation rate can reach more than $75 \%$ [3]. To further improve the performance of the damper, an elastic damper and a three-parameter fluid viscous damper can be installed in parallel. The main function of the elastic damper is to reduce the fundamental frequency of the system, to obtain better vibration isolation performance in the high-frequency band [4]. Besides, a three-parameter fluid viscous damper can also be combined with an active vibration isolator to realize hybrid vibration isolation, which will increase the performance and reliability of the system $[5][6]$.

The modeling methods of a three-parameter fluid viscous damper can be divided into two categories, one belongs to lumped parameter modeling method. The other belongs to the approximate analytical method. The lumped parameter modeling method is very simple in form and can also consider the influence of nonlinear damping, but it can not establish the relationship with the specific design parameters of the damper, so it can only be used for the design of stiffness coefficient and damping coefficient [2][3][7]. The approximate analytical method can establish the relationship with the specific design parameters of the damper [9][10][11]. The three-parameter fluid viscous damper uses the damping produced when the fluid flows through the damping orifice to mitigate the vibration energy. Due to the special configuration of damping orifice [12], the fluid will produce a contraction effect and expansion effect when flowing through the damping orifice, which will cause nonlinear damping and stiffness. For the convenience of modeling, most scholars did not consider the influence of nonlinear characteristics when using approximate analytical methods [3][4][8][11]. In describing the flow state in the damping orifice, they used the uncorrected hydraulic resistance, therefore the damping and hydraulic obtained are linear [13]. Shi [9] linearized the nonlinear damping according to the energy consumption, which is a linearized modeling method. Jiao's research points out that the reason for the nonlinear stiffness 
and damping of this damper is that the corrected hydraulic resistance is used when considering the contraction and expansion effect of damping orifice, and the nonlinear velocity term in the corrected hydraulic resistance leads to the nonlinear stiffness and damping [14]. The linear model is suitable for high viscosity fluid and the damping orifice has a large length-diameter ratio, but it is not accurate for low viscosity fluid. However, the influence of the principal stiffness of bellows was not considered in his research, so it is impossible to evaluate the influence of nonlinear stiffness and damping on vibration isolation performance.

In general, there are still some unsolved problems in the researches of nonlinear characteristics of the three-parameter fluid viscous damper. Most scholars have adopted the linearization model, ignoring the influence of nonlinear stiffness and damping, and ignoring the effect of principal stiffness when considering the nonlinear behavior of this damper [15], so it is unable to accurately evaluate the influence of nonlinear characteristics on this damper. In this paper, an approximate analytical modeling method is proposed to establish a nonlinear dynamic model of the three-parameter fluid viscous damper. In this model, the principal stiffness of bellows, the contraction and expansion effect of the damping orifice are considered. The nonlinear dynamic characteristics of the damper under sine force excitation are analyzed. At the same time, the differences between the linear model and the nonlinear model in predicting the performance of the damper are compared. The results show that the proposed model can be used to analyze the influence of nonlinear characteristics on the performance of the damper. 


\section{Modeling of three-parameter fluid viscous damper under displacement excitation and force excitation}

The three-parameter fluid viscous damper can work in force excitation mode and displacement excitation mode. In this section, the model of these two modes will be derived.

\subsection{Dynamic modeling of the damper under displacement excitation mode}

The three-parameter fluid viscous damper can be described in Fig.1(a). The main components of the damper consist of two bellows and a damping piston. Bellows have two functions, one is to provide principal stiffness, the other is to accommodate silicone oil. The damping piston is used to produce damping. Micro-vibrations have small amplitude, usually in the range of $10^{-6} \mathrm{~g}$ to $10^{-3} \mathrm{~g}$ [16]. So the displacement amplitude of micro-vibration will be very small. Under the small displacement excitation, the displacement amplitude of the damping piston will be very small. At this time, the volume change of the cavity is very limited. Before modeling, we assumed that the volume of the cavity would not change, and the change of internal pressure is also limited. Therefore, this paper does not consider the fluid-structure coupling effect between bellows and fluid. The effective diameter of the bellows should be used to calculate the internal pressure of the fluid-filled bellows [17]. Therefore, the bellows can be replaced by a single tube, and the area of cross-section is equal to the effective area of the bellows. In addition, the bellows also plays the role of providing stiffness, so the spring element can be used to replace the stiffness of bellows. In this way, the damper can be simplified by a single tube fluid viscous damper with two springs connected to the damping piston. The simplified model is shown in Fig.1(c). According to the Standards of the Expansion Joint Manufacturers Association standard [17], The 
effective area of the bellows can be calculated by the average diameter, and the calculation equation of the average diameter is

$$
D_{m}=D_{i n}+h+m_{c} \delta
$$

$D_{m}$ denotes the average diameter of bellows. $D_{i n}$ denotes the inner diameter of the straight section of bellows, the value is $25 \mathrm{~mm}$. $h$ denotes the height of bellows, the value is $6.3 \mathrm{~mm} . m_{c}$ denotes the number of layers of bellows with thickness $\delta$, because the bellows used in this paper are single-layer bellows, $m_{c}=1 . \delta$ denotes the thickness of bellows, the value is $0.2 \mathrm{~mm}$. After calculation, the average diameter of the bellows is $31.5 \mathrm{~mm}$.
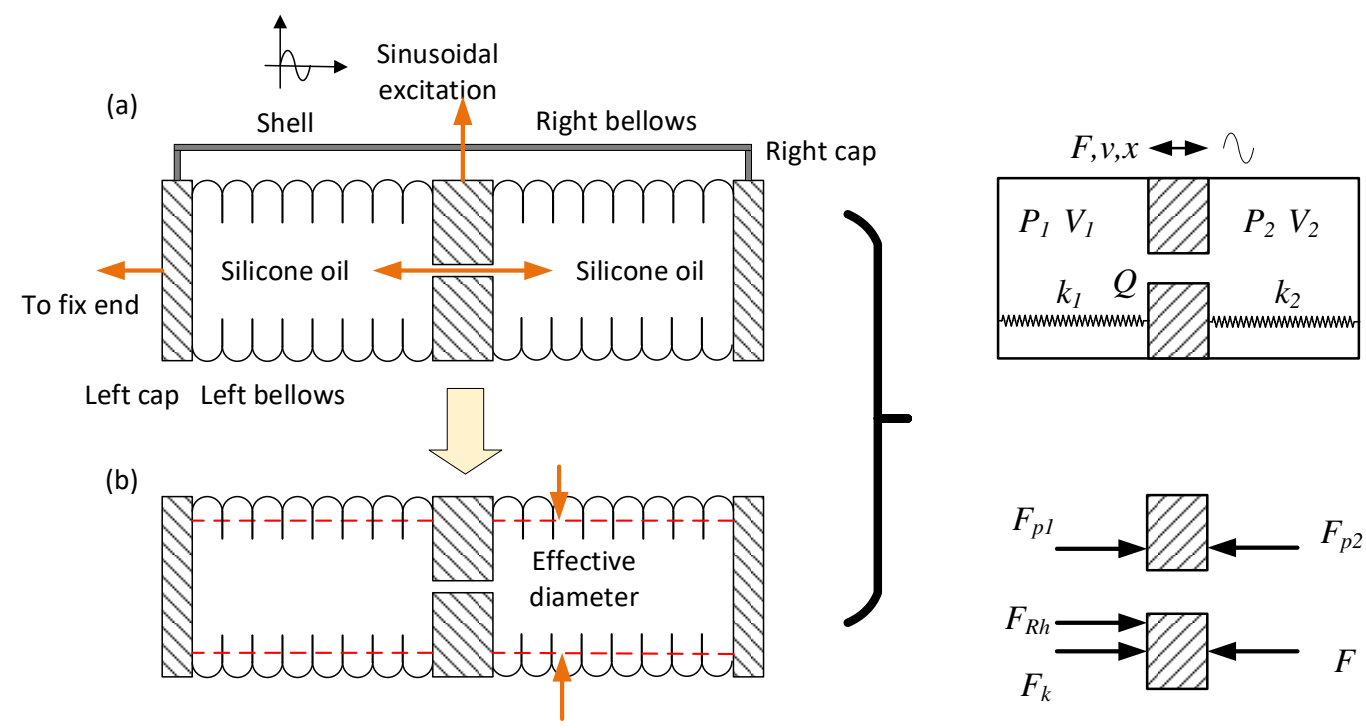

(c)

(d)

Fig.1 Simplified model of a three-parameter fluid viscous damper

By analyzing the force on the damping piston, the force balance equation can be written as

$$
\begin{gathered}
M \dot{v}+\left(P_{1}-P_{2}\right)\left(A_{p}-A_{g}\right)+R_{h} A_{g} Q=F \\
F-M \dot{v}-F_{\Delta p}-F_{k}-R_{h} A_{g} Q=0 \\
F_{k}=2 k_{1} x
\end{gathered}
$$

$M$ denotes the mass of the damping piston. $v$ denotes the velocity of the damping piston. $P_{1}$ denotes pressure in the left chamber. $P_{2}$ denotes pressure in the right 
chamber. $A_{p}$ denotes the effective area of bellows. $A_{g}$ denotes the area of the crosssection of the damping orifice. $R_{h}$ denotes hydraulic resistance. $Q$ denotes flow rate. $F$ denotes external force acting on the damping piston. $F_{\Delta p}$ denotes pressure differential force on the damping piston. $F_{k}$ denotes elastic force due to the compression of bellows. In Fig.1 (c), $V_{1}$ denotes the volume of the left chamber. $V_{2}$ denotes the volume of the right volume chamber. $k_{1}$ denotes equivalent stiffness of left bellows. $k_{2}$ denotes equivalent stiffness of right bellows. $F_{p 1}$ denotes pressure on the left damping piston. $F_{p 2}$ denotes pressure on the right damping piston. $F_{R h}$ denotes pressure differential force acting on the liquid column, $F_{R h}=R_{h} A_{g} Q$. Because the liquid column and the damping orifice interact through friction, $F_{R h}$ will also act on the damping piston. The three-parameter fluid viscous damper can work in displacement excitation mode, or force excitation mode. When the damping piston moves under the displacement excitation, the displacement amplitude of the damping piston does not change, but under different frequencies, the force required to produce the same amplitude displacement is quite different. This is mainly because the external force acting on the damping piston needs to overcome the pressure differential force inside the damper. This pressure differential force changes with frequencies [14], which leads to the changing external force. For constant amplitude force excitation, the displacement of the damping piston will vary with frequencies. This is mainly due to the difference in pressure at different frequencies. Under low-frequency excitation, the internal pressure of the damper is very small, part of the external excitation force is used to overcome the pressure differential force, and the other part is used to overcome the elastic force of the bellows. At this time, the damping piston has small displacement. In the high-frequency domain, the internal pressure increases obviously, and almost all 
external excitation force needs to be used to overcome the pressure inside the damper, so the displacement of the damping piston will decrease.

By analyzing the forces acting on the three-parameter fluid viscous damper, it can be found that the forces transmitted to the base include the pressure differential force and the elastic force of the spring. The pressure differential force can be written as

$$
F_{\Delta P}=\left(P_{1}-P_{2}\right) A_{p}
$$

According to the conservation of flow, the next equation can be obtained.

$$
\left\{\begin{array}{l}
-Q+A_{p} v=V_{1} \dot{P} / \beta \\
Q-A_{p} v=V_{2} \dot{P}_{2} / \beta
\end{array}\right.
$$

$\beta$ denotes bulk modulus of silicone oil. According to the force balance equation of the liquid column in the damping orifice, the following equation can be obtained

$$
\left\{\begin{array}{l}
\rho L_{g} \dot{Q}+R_{h} Q A_{g}=\left(P_{1}-P_{2}\right) A_{g} \\
R_{h}^{\prime}=c^{\prime} \frac{128 \mu L_{g}}{\pi d^{4}}
\end{array}\right.
$$

$\rho$ denotes the density of the fluid. $R_{h}^{\prime}$ denotes hydraulic resistance with consideration of contraction and expansion effect of damping orifice. $c^{\prime}$ denotes correction factor. $\mu$ denote viscosity of the fluid. $L_{g}$ denotes the length of the damping orifice. $d$ denotes the diameter of the damping orifice. The sharp edge damping orifice is used in this paper, according to Langhaar's experiment [18], the correction hydraulic resistance can be written as

$$
\begin{gathered}
\quad R_{h}^{\prime}=R_{h}\left[1+\left(\xi+\zeta_{1}+\zeta_{2}-2 m^{2} \beta_{2}\right) \frac{\operatorname{Re}}{64}\left(\frac{d}{L_{g}}\right)\right]=R_{h}\left[1+\left(\xi+c_{\zeta}\right) \frac{\operatorname{Re}}{64}\left(\frac{d}{L_{g}}\right)\right] \\
c_{\zeta}=\zeta_{1}+\zeta_{2}-2 m^{2} \beta_{2}, \quad \zeta_{1} \text { denotes the resistance coefficient of the inlet, } \zeta_{2}
\end{gathered}
$$

denotes the resistance coefficient due to the jet-flow. Re denotes Reynolds number. When $\operatorname{Re} \leq 30, \zeta=\zeta_{1}+\zeta_{2}=0.042$, when $\operatorname{Re}>2000, \zeta=\zeta_{1}+\zeta_{2}=0.49$. When the Reynolds number is between 30 and 2000, $\zeta$ changes linearly. $m$ denotes the 
diameter ratio, $m=d / D, D$ denotes the diameter of the damper. $\beta_{2}$ denotes the momentum correction coefficient at the outlet of the damping orifice. $\xi$ denotes correction factor. According to Langhaar's experiment [18], $\xi$ can be written as

$$
\begin{aligned}
& \left\{\begin{array}{l}
\xi=1+2.62\left(L_{g} / \operatorname{Re} d\right)^{1 / 4}, L_{g} / \operatorname{Re} d \leq 0.058 \\
\xi=2.28, L_{g} / \operatorname{Re} d>0.058
\end{array}\right. \\
& R_{h}^{\prime}=\left\{\begin{array}{l}
\gamma_{1} R_{h}, L_{g} / \operatorname{Re} d \leq 0.058 \\
\gamma_{2} R_{h}, L_{g} / \operatorname{Re} d>0.058
\end{array}\right. \\
& \gamma_{1}=1+\tau_{1} v^{3 / 4}+\tau_{2} v, \gamma_{2}=1+\tau_{3} v, \quad \tau_{1}=\frac{2.62}{64}\left(\frac{\rho}{\mu L_{g}}\right)^{\frac{3}{4}} d^{\frac{3}{2}} d_{\text {ratio }}^{\frac{3}{4}} \\
& \tau_{2}=\left(1+c_{\zeta}\right) \frac{\rho d^{2}}{64 \mu L_{g}} d_{\text {ratio }}, \tau_{3}=\left(2.28+c_{\zeta}\right) \frac{\rho d^{2}}{64 \mu L_{g}} d_{\text {ratio }}, d_{\text {ratio }}=d / D
\end{aligned}
$$

It can be seen from the above equation that the corrected hydraulic resistance contains the first-order term of velocity, which indicates that the hydraulic resistance changes with the fluid velocity in the damping orifice.

Because the displacement of the damping piston is very small under the microvibration excitation, the volume of the cavity changes little, $V_{1}=V_{2}=V_{0}$. According to equations (5), (6), and (7), the transfer function of pressure differential force relative to displacement can be obtained.

\subsection{Dynamic modeling of the damper under force excitation}

When the damper working under the sinusoidal force excitation, the solution of the pressure differential force is still consistent with the previous. However, the displacement of the damping piston is needed when establishing the transfer function of pressure differential force relative to displacement. Sinusoidal excitation force can be written as

$$
F=F_{0} \sin \omega t
$$


$F_{0}$ denotes the amplitude of sinusoidal force. $\omega$ denotes frequency. $t$ denotes time. By using Laplace transform, external force in the complex domain can be expressed as

$$
F(s)=M \frac{\left\{\begin{array}{l}
s^{4}+\left(R_{h} A_{g} / \rho L_{g}\right) s^{3}+\left[\begin{array}{l}
2 \beta A_{g} / \rho L_{g} V_{0}+ \\
2 \beta A_{P}\left(A_{P}-A_{g}\right) / M V_{0}+k / M
\end{array}\right] s^{2}+ \\
\left(\begin{array}{l}
2 \beta R_{h} A_{g} A_{P}^{2} / M \rho L_{g} V_{0}+ \\
R_{h} A_{g} k / M \rho L_{g}
\end{array}\right) s+2 \beta A_{g} k / M \rho L_{g} V_{0}
\end{array}\right\}}{s^{2}+R_{h} A_{g} / \rho L_{g} s+2 \beta A_{g} / \rho L_{g} V_{0}} X(s)
$$

Flow rate $Q$ can be written as

$$
Q(s)=\frac{2 \beta A_{p} A_{g} s}{\rho L_{g} V_{0} s^{2}+R_{h} A_{g} V_{0} s+2 \beta A_{g}} X(s)
$$

According to equations (2) and (3), the transfer function can be obtained.

$$
\begin{gathered}
\frac{F_{\Delta P}(s)}{F(s)}=G \frac{s^{2}+A_{1} s}{s^{4}+A_{1} s^{3}+A_{2} s^{2}+A_{3} s+A_{4}} \\
A_{1}=\frac{R_{h} A_{g}}{\rho L_{g}}, A_{2}=\frac{2 \beta A_{g}}{\rho L_{g} V_{0}}+\frac{2 \beta A_{P}\left(A_{P}-A_{g}\right)}{M V_{0}}+\frac{k}{M}, A_{3}=\frac{2 \beta R_{h} A_{g} A_{P}^{2}}{M \rho L_{g} V_{0}}+\frac{R_{h} A_{g} k}{M \rho L_{g}} \\
A_{4}=\frac{2 \beta A_{g} k}{M \rho L_{g} V_{0}}, A_{5}=\frac{2 \beta A_{g}}{\rho L_{g} V_{0}}, G=\frac{2 \beta A_{P}\left(A_{P}-A_{g}\right)}{M V_{0}}
\end{gathered}
$$

Pressure differential force in the complex domain can be written as

$$
F_{\Delta P}(s)=\frac{G F_{0} \omega}{s^{2}+\omega^{2}} \frac{s^{2}+A_{1} s}{s^{4}+A_{1} s^{3}+A_{2} s^{2}+A_{3} s+A_{4}}
$$

Displacement of damping piston in the complex domain can be written as

$$
X(s)=\frac{F_{0} \omega}{M} \frac{1}{s^{2}+\omega^{2}} \frac{s^{2}+A_{1} s+A_{5}}{s^{4}+A_{1} s^{3}+A_{2} s^{2}+A_{3} s+A_{4}}
$$

Make the denominator of equation (15) equal to zero to obtain characteristic roots.

$$
s^{4}+A_{1} s^{3}+A_{2} s^{2}+A_{3} s+A_{4}=0
$$

Characteristic equation (16) has four roots. Therefore, the pressure differential force will be different due to the different forms of characteristic roots. Characteristic roots of the equation (16) depending on the damping orifice diameter, length, and viscosity of silicone oil. According to the different forms of characteristic roots, pressure differential force can be divide into two cases to discuss. 
1) Equation (16) has a pair of unequal real roots and a pair of conjugate complex roots. The characteristic roots can be expressed as

$$
s_{1}=x_{1}, s_{2}=x_{2}, s_{3}=m_{1}+j n_{1}, s_{4}=m_{1}-j n_{1}
$$

Taking inverse Laplace to transform to the equation (15), pressure differential force in the time domain can be obtained.

$$
F_{\Delta P}(t)=G F_{0} \omega\left\{\begin{array}{l}
2 U \cos \omega t-2 V \sin \omega t+c_{1} e^{x_{1} t}+c_{2} e^{x_{3} t}+ \\
2 e^{m_{1} t}\left(X \cos n_{1} t-Y \sin n_{1} t\right)
\end{array}\right\}
$$

$$
\begin{aligned}
& B_{1}=2\left[\omega\left(x_{1}+x_{2}\right)\left(m_{1}^{2}+n_{1}^{2}-\omega^{2}\right)+2 m_{1} \omega\left(x_{1} x_{2}-\omega^{2}\right)\right] \\
& B_{2}=2\left[-2 m_{1} \omega^{2}\left(x_{1}+x_{2}\right)+\left(x_{1} x_{2}-\omega^{2}\right)\left(m_{1}^{2}+n_{1}^{2}-\omega^{2}\right)\right] \\
& B_{3}=-\omega, B_{4}=A_{1}, U=\left(B_{1} B_{3}+B_{2} B_{4}\right) /\left(B_{1}^{2}+B_{2}^{2}\right), V=\left(B_{1} B_{4}-B_{2} B_{3}\right) /\left(B_{1}^{2}+B_{2}^{2}\right) \\
& c_{1}=\frac{x_{1}^{2}+A_{1} x_{1}}{\left(x_{1}-x_{2}\right)\left(x_{1}^{2}+\omega^{2}\right)\left[\left(x_{1}-m_{1}\right)^{2}+n_{1}^{2}\right]}, c_{2}=\frac{x_{2}^{2}+A_{1} x_{2}}{\left(x_{2}-x_{1}\right)\left(x_{2}^{2}+\omega^{2}\right)\left[\left(x_{2}-m_{1}\right)^{2}+n_{1}^{2}\right]} \\
& D_{1}=-2 n_{1}^{2}\left\{\left(m_{1}^{2}-n_{1}^{2}+\omega^{2}\right)\left(2 m_{1}-x_{1}-x_{2}\right)+2 m_{1}\left[\left(m_{1}-x_{1}\right)\left(m_{1}-x_{2}\right)-n_{1}^{2}\right]\right\} \\
& D_{2}=2 n_{1}\left\{\left(m_{1}^{2}-n_{1}^{2}+\omega^{2}\right)\left[\left(m_{1}-x_{1}\right)\left(m_{1}-x_{2}\right)-n_{1}^{2}\right]-2 m_{1} n_{1}^{2}\left(2 m_{1}-x_{1}-x_{2}\right)\right\} \\
& D_{3}=m_{1}^{2}-n_{1}^{2}+A_{1} m_{1}, D_{4}=2 m_{1} n_{1}+A_{1} n_{1} \\
& X=\left(D_{1} D_{3}+D_{2} D_{4}\right) /\left(D_{1}^{2}+D_{2}^{2}\right), Y=\left(D_{1} D_{4}-D_{2} D_{3}\right) /\left(D_{1}^{2}+D_{2}^{2}\right)
\end{aligned}
$$

When $L_{g} / \operatorname{Re} d \leq 0.058$, because the hydraulic resistance contains the nonlinear term of velocity, the pressure differential force contains the nonlinear component. Nonlinear damping force can be written as

$$
\begin{aligned}
& F_{c}(t)=2 G F_{0} U \omega \cos \omega t\left\{4 \omega^{2}\left[m_{2}\left(x_{3}^{2}-\omega^{2}+y_{3}^{2}\right)+x_{3}\left(m_{2}^{2}-\omega^{2}+n_{2}^{2}\right)\right]\right\} \cdot(-\omega)+ \\
&=\frac{2 G F_{0}}{x_{0}} \cdot \frac{\left\{2 \omega\left[\left(x_{3}^{2}-\omega^{2}+y_{3}^{2}\right)\left(m_{2}^{2}-\omega^{2}+n_{2}^{2}\right)-4 m_{2} x_{3} \omega^{2}\right]\right\} \cdot\left(\frac{R_{h}^{\prime} A_{g}}{\rho L_{g}}\right)}{\left\{2\left[\omega\left(x_{1}+x_{2}\right)\left(m_{1}^{2}+n_{1}^{2}-\omega^{2}\right)+2 m_{1} \omega\left(x_{1} x_{2}-\omega^{2}\right)\right]\right\}^{2}+} v \\
&\left\{2\left[-2 m_{1} \omega^{2}\left(x_{1}+x_{2}\right)+\left(x_{1} x_{2}-\omega^{2}\right)\left(m_{1}^{2}+n_{1}^{2}-\omega^{2}\right)\right]\right\}^{2}
\end{aligned}
$$




$$
\begin{aligned}
& =\frac{2 G F_{0}}{x_{0}} \cdot \frac{\left\{4 \omega^{2}\left[m_{2}\left(x_{3}{ }^{2}-\omega^{2}+y_{3}{ }^{2}\right)+x_{3}\left(m_{2}{ }^{2}-\omega^{2}+n_{2}{ }^{2}\right)\right]\right\} \cdot(-\omega)}{\left\{2\left[\omega\left(x_{1}+x_{2}\right)\left(m_{1}{ }^{2}+n_{1}{ }^{2}-\omega^{2}\right)+2 m_{1} \omega\left(x_{1} x_{2}-\omega^{2}\right)\right]\right\}^{2}+} v \\
& \left\{2\left[-2 m_{1} \omega^{2}\left(x_{1}+x_{2}\right)+\left(x_{1} x_{2}-\omega^{2}\right)\left(m_{1}^{2}+n_{1}{ }^{2}-\omega^{2}\right)\right]\right\}^{2} \\
& +\frac{\left\{2 \omega\left[\left(x_{3}{ }^{2}-\omega^{2}+y_{3}{ }^{2}\right)\left(m_{2}{ }^{2}-\omega^{2}+n_{2}{ }^{2}\right)-4 m_{2} x_{3} \omega^{2}\right]\right\} \cdot\left(\frac{A_{g}}{\rho L_{g}}\right)}{\left\{2\left[\omega\left(x_{1}+x_{2}\right)\left(m_{1}{ }^{2}+n_{1}{ }^{2}-\omega^{2}\right)+2 m_{1} \omega\left(x_{1} x_{2}-\omega^{2}\right)\right]\right\}^{2}+} R_{h}^{\prime} v \\
& \left\{2\left[-2 m_{1} \omega^{2}\left(x_{1}+x_{2}\right)+\left(x_{1} x_{2}-\omega^{2}\right)\left(m_{1}^{2}+n_{1}^{2}-\omega^{2}\right)\right]\right\}^{2} \\
& =\lambda_{1} v+\lambda_{2} R_{h}^{\prime} v \\
& =\lambda_{1} v+\lambda_{2}\left(1+\tau_{1} v^{3 / 4}+\tau_{2} v\right) R_{h} v \\
& \lambda_{1}=\frac{2 G F_{0}}{x_{0}} \cdot \frac{\left\{4 \omega^{2}\left[m_{2}\left(x_{3}{ }^{2}-\omega^{2}+y_{3}{ }^{2}\right)+x_{3}\left(m_{2}{ }^{2}-\omega^{2}+n_{2}{ }^{2}\right)\right]\right\} \cdot(-\omega)}{\left\{2\left[\omega\left(x_{1}+x_{2}\right)\left(m_{1}{ }^{2}+n_{1}{ }^{2}-\omega^{2}\right)+2 m_{1} \omega\left(x_{1} x_{2}-\omega^{2}\right)\right]\right\}^{2}+} \\
& \left\{2\left[-2 m_{1} \omega^{2}\left(x_{1}+x_{2}\right)+\left(x_{1} x_{2}-\omega^{2}\right)\left(m_{1}^{2}+n_{1}^{2}-\omega^{2}\right)\right]\right\}^{2} \\
& \lambda_{2}=\frac{\left\{2 \omega\left[\left(x_{3}^{2}-\omega^{2}+y_{3}^{2}\right)\left(m_{2}^{2}-\omega^{2}+n_{2}^{2}\right)-4 m_{2} x_{3} \omega^{2}\right]\right\} \cdot\left(\frac{A_{g}}{\rho L_{g}}\right)}{\left\{2\left[\omega\left(x_{1}+x_{2}\right)\left(m_{1}^{2}+n_{1}^{2}-\omega^{2}\right)+2 m_{1} \omega\left(x_{1} x_{2}-\omega^{2}\right)\right]\right\}^{2}+} \\
& \left\{2\left[-2 m_{1} \omega^{2}\left(x_{1}+x_{2}\right)+\left(x_{1} x_{2}-\omega^{2}\right)\left(m_{1}^{2}+n_{1}^{2}-\omega^{2}\right)\right]\right\}^{2}
\end{aligned}
$$

The nonlinear elastic force can be written as

$$
\begin{aligned}
& F_{k}(t)=-2 G F_{0} V \omega \sin \omega t \\
& \left\{2\left[\omega\left(x_{1}+x_{2}\right)\left(m_{1}^{2}+n_{1}^{2}-\omega^{2}\right)+2 m_{1} \omega\left(x_{1} x_{2}-\omega^{2}\right)\right]\right\} \cdot\left(\frac{R_{h}^{\prime} A_{g}}{\rho L_{g}}\right)- \\
& =\frac{-2 G F_{0} \omega}{x_{0}} \cdot \frac{\left\{2\left[-2 m_{1} \omega^{2}\left(x_{1}+x_{2}\right)+\left(x_{1} x_{2}-\omega^{2}\right)\left(m_{1}^{2}+n_{1}^{2}-\omega^{2}\right)\right]\right\} \cdot(-\omega)}{\left\{2\left[\omega\left(x_{1}+x_{2}\right)\left(m_{1}^{2}+n_{1}^{2}-\omega^{2}\right)+2 m_{1} \omega\left(x_{1} x_{2}-\omega^{2}\right)\right]\right\}^{2}+} x \\
& \left\{2\left[-2 m_{1} \omega^{2}\left(x_{1}+x_{2}\right)+\left(x_{1} x_{2}-\omega^{2}\right)\left(m_{1}^{2}+n_{1}^{2}-\omega^{2}\right)\right]\right\}^{2}
\end{aligned}
$$




$$
\begin{aligned}
& =\frac{-2 G F_{0} \omega}{x_{0}} \frac{\left\{2\left[\omega\left(x_{1}+x_{2}\right)\left(m_{1}^{2}+n_{1}^{2}-\omega^{2}\right)+2 m_{1} \omega\left(x_{1} x_{2}-\omega^{2}\right)\right]\right\} \cdot\left(\frac{A_{g}}{\rho L_{g}}\right)}{\left\{2\left[\omega\left(x_{1}+x_{2}\right)\left(m_{1}^{2}+n_{1}^{2}-\omega^{2}\right)+2 m_{1} \omega\left(x_{1} x_{2}-\omega^{2}\right)\right]\right\}^{2}+} R_{h}^{\prime} x \\
& \left\{2\left[-2 m_{1} \omega^{2}\left(x_{1}+x_{2}\right)+\left(x_{1} x_{2}-\omega^{2}\right)\left(m_{1}^{2}+n_{1}^{2}-\omega^{2}\right)\right]\right\}^{2} \\
& +\frac{-2 G F_{0} \omega}{x_{0}} \cdot \frac{\left\{2\left[-2 m_{1} \omega^{2}\left(x_{1}+x_{2}\right)+\left(x_{1} x_{2}-\omega^{2}\right)\left(m_{1}^{2}+n_{1}^{2}-\omega^{2}\right)\right]\right\} \cdot(-\omega)}{\left\{2\left[\omega\left(x_{1}+x_{2}\right)\left(m_{1}^{2}+n_{1}^{2}-\omega^{2}\right)+2 m_{1} \omega\left(x_{1} x_{2}-\omega^{2}\right)\right]\right\}^{2}+} x \\
& \left\{2\left[-2 m_{1} \omega^{2}\left(x_{1}+x_{2}\right)+\left(x_{1} x_{2}-\omega^{2}\right)\left(m_{1}^{2}+n_{1}^{2}-\omega^{2}\right)\right]\right\}^{2} \\
& =\lambda_{3} R_{h}^{\prime} x+\lambda_{4} x=\lambda_{3} R_{h}\left(1+\tau_{1} v^{3 / 4}+\tau_{2} v\right) x+\lambda_{4} x \\
& \lambda_{3}=\frac{-2 G F_{0} \omega}{x_{0}} \frac{\left\{2\left[\omega\left(x_{1}+x_{2}\right)\left(m_{1}^{2}+n_{1}^{2}-\omega^{2}\right)+2 m_{1} \omega\left(x_{1} x_{2}-\omega^{2}\right)\right]\right\} \cdot\left(\frac{A_{g}}{\rho L_{g}}\right)}{\left\{2\left[\omega\left(x_{1}+x_{2}\right)\left(m_{1}^{2}+n_{1}^{2}-\omega^{2}\right)+2 m_{1} \omega\left(x_{1} x_{2}-\omega^{2}\right)\right]\right\}^{2}+} \\
& \left\{2\left[-2 m_{1} \omega^{2}\left(x_{1}+x_{2}\right)+\left(x_{1} x_{2}-\omega^{2}\right)\left(m_{1}^{2}+n_{1}^{2}-\omega^{2}\right)\right]\right\}^{2} \\
& \lambda_{4}=\frac{-2 G F_{0} \omega}{x_{0}} \cdot \frac{\left\{2\left[-2 m_{1} \omega^{2}\left(x_{1}+x_{2}\right)+\left(x_{1} x_{2}-\omega^{2}\right)\left(m_{1}^{2}+n_{1}^{2}-\omega^{2}\right)\right]\right\} \cdot(-\omega)}{\left\{2\left[\omega\left(x_{1}+x_{2}\right)\left(m_{1}^{2}+n_{1}^{2}-\omega^{2}\right)+2 m_{1} \omega\left(x_{1} x_{2}-\omega^{2}\right)\right]\right\}^{2}+} \\
& \left\{2\left[-2 m_{1} \omega^{2}\left(x_{1}+x_{2}\right)+\left(x_{1} x_{2}-\omega^{2}\right)\left(m_{1}^{2}+n_{1}^{2}-\omega^{2}\right)\right]\right\}^{2}
\end{aligned}
$$

When $L_{g} / \operatorname{Re} d>0.058$, the nonlinear damping force can be written as

$$
\begin{aligned}
& F_{c}(t)=2 G F_{0} U \omega \cos \omega t\left\{4 \omega^{2}\left[m_{2}\left(x_{3}{ }^{2}-\omega^{2}+y_{3}{ }^{2}\right)+x_{3}\left(m_{2}{ }^{2}-\omega^{2}+n_{2}{ }^{2}\right)\right]\right\} \cdot(-\omega)+ \\
&=\frac{2 G F_{0}}{x_{0}} \cdot \frac{\left\{2 \omega\left[\left(x_{3}{ }^{2}-\omega^{2}+y_{3}{ }^{2}\right)\left(m_{2}{ }^{2}-\omega^{2}+n_{2}{ }^{2}\right)-4 m_{2} x_{3} \omega^{2}\right]\right\} \cdot\left(\frac{R_{h}^{\prime} A_{g}}{\rho L_{g}}\right)}{\left\{2\left[\omega\left(x_{1}+x_{2}\right)\left(m_{1}{ }^{2}+n_{1}{ }^{2}-\omega^{2}\right)+2 m_{1} \omega\left(x_{1} x_{2}-\omega^{2}\right)\right]\right\}^{2}+} v \\
&\left\{2\left[-2 m_{1} \omega^{2}\left(x_{1}+x_{2}\right)+\left(x_{1} x_{2}-\omega^{2}\right)\left(m_{1}{ }^{2}+n_{1}{ }^{2}-\omega^{2}\right)\right]\right\}^{2}
\end{aligned}
$$




$$
\begin{aligned}
& =\frac{2 G F_{0}}{x_{0}} \cdot \frac{\left\{4 \omega^{2}\left[m_{2}\left(x_{3}^{2}-\omega^{2}+y_{3}^{2}\right)+x_{3}\left(m_{2}^{2}-\omega^{2}+n_{2}^{2}\right)\right]\right\} \cdot(-\omega)}{\left\{2\left[\omega\left(x_{1}+x_{2}\right)\left(m_{1}^{2}+n_{1}^{2}-\omega^{2}\right)+2 m_{1} \omega\left(x_{1} x_{2}-\omega^{2}\right)\right]\right\}^{2}+} v \\
& \left\{2\left[-2 m_{1} \omega^{2}\left(x_{1}+x_{2}\right)+\left(x_{1} x_{2}-\omega^{2}\right)\left(m_{1}^{2}+n_{1}^{2}-\omega^{2}\right)\right]\right\}^{2} \\
& +\frac{2 G F_{0}}{x_{0}} \cdot \frac{\left\{2 \omega\left[\left(x_{3}{ }^{2}-\omega^{2}+y_{3}^{2}\right)\left(m_{2}{ }^{2}-\omega^{2}+n_{2}{ }^{2}\right)-4 m_{2} x_{3} \omega^{2}\right]\right\} \cdot\left(\frac{A_{g}}{\rho L_{g}}\right)}{\left\{2\left[\omega\left(x_{1}+x_{2}\right)\left(m_{1}{ }^{2}+n_{1}{ }^{2}-\omega^{2}\right)+2 m_{1} \omega\left(x_{1} x_{2}-\omega^{2}\right)\right]\right\}^{2}+} R_{h}^{\prime} v \\
& \left\{2\left[-2 m_{1} \omega^{2}\left(x_{1}+x_{2}\right)+\left(x_{1} x_{2}-\omega^{2}\right)\left(m_{1}{ }^{2}+n_{1}{ }^{2}-\omega^{2}\right)\right]\right\}^{2} \\
& =\lambda_{5} v+\lambda_{6} R_{h}^{\prime} v=\lambda_{5} v+\lambda_{6} R_{h}\left(1+\tau_{3} v\right) \\
& \lambda_{5}=\frac{2 G F_{0}}{x_{0}} \cdot \frac{\left\{4 \omega^{2}\left[m_{2}\left(x_{3}^{2}-\omega^{2}+y_{3}^{2}\right)+x_{3}\left(m_{2}^{2}-\omega^{2}+n_{2}^{2}\right)\right]\right\} \cdot(-\omega)}{\left\{2\left[\omega\left(x_{1}+x_{2}\right)\left(m_{1}^{2}+n_{1}^{2}-\omega^{2}\right)+2 m_{1} \omega\left(x_{1} x_{2}-\omega^{2}\right)\right]\right\}^{2}+} \\
& \left\{2\left[-2 m_{1} \omega^{2}\left(x_{1}+x_{2}\right)+\left(x_{1} x_{2}-\omega^{2}\right)\left(m_{1}^{2}+n_{1}^{2}-\omega^{2}\right)\right]\right\}^{2} \\
& \lambda_{6}=\frac{2 G F_{0}}{x_{0}} \cdot \frac{\left\{2 \omega\left[\left(x_{3}^{2}-\omega^{2}+y_{3}^{2}\right)\left(m_{2}^{2}-\omega^{2}+n_{2}^{2}\right)-4 m_{2} x_{3} \omega^{2}\right]\right\} \cdot\left(\frac{A_{g}}{\rho L_{g}}\right)}{\left\{2\left[\omega\left(x_{1}+x_{2}\right)\left(m_{1}^{2}+n_{1}^{2}-\omega^{2}\right)+2 m_{1} \omega\left(x_{1} x_{2}-\omega^{2}\right)\right]\right\}^{2}+} \\
& \left\{2\left[-2 m_{1} \omega^{2}\left(x_{1}+x_{2}\right)+\left(x_{1} x_{2}-\omega^{2}\right)\left(m_{1}^{2}+n_{1}^{2}-\omega^{2}\right)\right]\right\}^{2}
\end{aligned}
$$

The nonlinear elastic force can be written as

$$
F_{k}(t)=-2 G F_{0} V \omega \sin \omega t
$$

$$
\begin{aligned}
&\left\{2\left[\omega\left(x_{1}+x_{2}\right)\left(m_{1}^{2}+n_{1}^{2}-\omega^{2}\right)+2 m_{1} \omega\left(x_{1} x_{2}-\omega^{2}\right)\right]\right\} \cdot\left(\frac{R_{h}^{\prime} A_{g}}{\rho L_{g}}\right)- \\
&=\frac{-2 G F_{0} \omega}{x_{0}} \cdot \frac{\left\{2\left[-2 m_{1} \omega^{2}\left(x_{1}+x_{2}\right)+\left(x_{1} x_{2}-\omega^{2}\right)\left(m_{1}^{2}+n_{1}^{2}-\omega^{2}\right)\right]\right\} \cdot(-\omega)}{\left\{2\left[\omega\left(x_{1}+x_{2}\right)\left(m_{1}^{2}+n_{1}^{2}-\omega^{2}\right)+2 m_{1} \omega\left(x_{1} x_{2}-\omega^{2}\right)\right]\right\}^{2}+} x \\
&\left\{2\left[-2 m_{1} \omega^{2}\left(x_{1}+x_{2}\right)+\left(x_{1} x_{2}-\omega^{2}\right)\left(m_{1}^{2}+n_{1}^{2}-\omega^{2}\right)\right]\right\}^{2}
\end{aligned}
$$




$$
\begin{gathered}
=\frac{-2 G F_{0} \omega}{x_{0}} \cdot \frac{\left\{2\left[\omega\left(x_{1}+x_{2}\right)\left(m_{1}^{2}+n_{1}^{2}-\omega^{2}\right)+2 m_{1} \omega\left(x_{1} x_{2}-\omega^{2}\right)\right]\right\} \cdot\left(\frac{A_{g}}{\rho L_{g}}\right)}{\left\{2\left[\omega\left(x_{1}+x_{2}\right)\left(m_{1}^{2}+n_{1}^{2}-\omega^{2}\right)+2 m_{1} \omega\left(x_{1} x_{2}-\omega^{2}\right)\right]\right\}^{2}+} R_{h}^{\prime} x \\
+\frac{-2 G F_{0} \omega}{x_{0}} \cdot \frac{\left\{2\left[-2 m_{1} \omega^{2}\left(x_{1}+x_{2}\right)+\left(x_{1} x_{2}-\omega^{2}\right)\left(m_{1}^{2}+n_{1}^{2}-\omega^{2}\right)\right]\right\} \cdot(-\omega)}{\left\{2\left[\omega\left(x_{1}+x_{2}\right)\left(m_{1}^{2}+n_{1}^{2}-\omega^{2}\right)+2 m_{1} \omega\left(x_{1} x_{2}-\omega^{2}\right)\right]\right\}^{2}+} x \\
\lambda_{7}=\frac{-2 G F_{0} \omega}{x_{0}} \cdot \frac{\left\{2\left[\omega\left(x_{1}+x_{2}\right)\left(m_{1}^{2}+n_{1}^{2}-\omega^{2}\right)+2 m_{1} \omega\left(x_{1} x_{2}-\omega^{2}\right)\right]\right\} \cdot\left(\frac{A_{g}}{\rho L_{g}}\right)}{\left\{2\left[\omega\left(x_{1}+x_{2}\right)\left(m_{1}^{2}+n_{1}^{2}-\omega^{2}\right)+2 m_{1} \omega\left(x_{1} x_{2}-\omega^{2}\right)\right]\right\}^{2}+} \\
\left.\left.\left.=\lambda_{7} R_{h}^{\prime} x+\lambda_{8} x=\lambda_{7}\left(1+\tau_{3} \nu\right) x+\lambda_{8} x_{1}^{2}-\omega^{2}\right)\right]\right\}^{2} \\
\lambda_{8}=\frac{-2 G F_{0} \omega}{x_{0}} \cdot \frac{\left\{2\left[-2 m_{1} \omega^{2}\left(x_{1}+x_{2}\right)+\left(x_{1} x_{2}-\omega^{2}\right)\left(m_{1}^{2}+n_{1}^{2}-\omega^{2}\right)\right]\right\} \cdot(-\omega)}{\left\{2\left[\omega\left(x_{1}+x_{2}\right)\left(m_{1}^{2}+n_{1}^{2}-\omega^{2}\right)+2 m_{1} \omega\left(x_{1} x_{2}-\omega^{2}\right)\right]\right\}^{2}+} \\
\left\{2\left[-2 m_{1} \omega^{2}\left(x_{1}+x_{2}\right)+\left(x_{1} x_{2}-\omega^{2}\right)\left(m_{1}^{2}+n_{1}^{2}-\omega^{2}\right)\right]\right\}^{2}
\end{gathered}
$$

2) Equation (16) has two pairs of conjugate complex roots. Characteristic roots can be expressed as

$$
s_{1}=x_{3}+\mathrm{j} y_{3}, s_{2}=x_{3}-\mathrm{j} y_{3}, s_{3}=m_{2}+\mathrm{j} n_{2}, s_{4}=m_{2}-\mathrm{j} n_{2}
$$

Taking inverse Laplace to transform to the equation (14), pressure differential force in the time domain can be obtained.

$$
\begin{gathered}
F_{\Delta P}(t)=2 G F_{0} \omega\left\{\begin{array}{l}
U \cos \omega t-V \sin \omega t+e^{x_{3} t}\left(M \cos y_{3} t-N \sin y_{3} t\right) \\
e^{m_{2} t}\left(X \cos n_{2} t-Y \sin n_{2} t\right)
\end{array}\right\} \\
B_{1}=4 \omega^{2}\left[m_{2}\left(x_{3}{ }^{2}-\omega^{2}+y_{3}{ }^{2}\right)+x_{3}\left(m_{2}{ }^{2}-\omega^{2}+n_{2}{ }^{2}\right)\right] \\
B_{2}=2 \omega\left[\left(x_{3}{ }^{2}-\omega^{2}+y_{3}{ }^{2}\right)\left(m_{2}{ }^{2}-\omega^{2}+n_{2}{ }^{2}\right)-4 m_{2} x_{3} \omega^{2}\right] \\
B_{3}=-\omega^{2}, B_{4}=\omega A_{1}, U=\left(B_{1} B_{3}+B_{2} B_{4}\right) /\left(B_{1}{ }^{2}+B_{2}{ }^{2}\right), V=\left(B_{1} B_{4}-B_{2} B_{3}\right) /\left(B_{1}{ }^{2}+B_{2}{ }^{2}\right) \\
D_{1}=-4 y_{3}{ }^{2}\left\{\left(x_{3}-m_{2}\right)\left(x_{3}{ }^{2}+\omega^{2}-y_{3}{ }^{2}\right)+x_{3}\left[\left(x_{3}-m_{2}\right)^{2}-y_{3}{ }^{2}+n_{2}{ }^{2}\right]\right\} \\
D_{2}=2 y_{3}\left\{\left(x_{3}{ }^{2}-y_{3}{ }^{2}+\omega^{2}\right)\left[\left(x_{3}-m_{2}\right)^{2}-y_{3}{ }^{2}+n_{2}{ }^{2}\right]-4 x_{3} y_{3}{ }^{2}\left(x_{3}-m_{2}\right)\right\}
\end{gathered}
$$




$$
\begin{aligned}
& D_{3}=x_{3}^{2}-y_{3}^{2}+A_{1} x_{3} \\
& D_{4}=2 x_{3} y_{3}+A_{1} y_{3} \\
& M=\left(D_{1} D_{3}+D_{2} D_{4}\right) /\left(D_{1}^{2}+D_{2}^{2}\right), N=\left(D_{1} D_{4}-D_{2} D_{3}\right) /\left(D_{1}^{2}+D_{2}^{2}\right) \\
& E_{1}=-4 n_{2}^{2}\left\{\left(m_{2}-x_{3}\right)\left(m_{2}^{2}-n_{2}^{2}+\omega^{2}\right)+m_{2}\left[\left(m_{2}-x_{3}\right)^{2}-n_{2}^{2}+y_{3}^{2}\right]\right\} \\
& E_{2}=2 n_{2}\left\{\left(m_{2}^{2}-n_{2}^{2}+\omega^{2}\right)\left[\left(m_{2}-x_{3}\right)^{2}-n_{2}^{2}+y_{3}^{2}\right]-4 m_{2} n_{2}^{2}\left(m_{2}-x_{3}\right)\right\} \\
& E_{3}=m_{2}^{2}-n_{2}^{2}+m_{2} A_{1} \\
& E_{4}=2 m_{2} n_{2}+n_{2} A_{1} \\
& X=\left(E_{1} E_{3}+E_{2} E_{4}\right) /\left(E_{1}^{2}+E_{2}^{2}\right), Y=\left(E_{1} E_{4}-E_{2} E_{3}\right) /\left(E_{1}^{2}+E_{2}^{2}\right)
\end{aligned}
$$

When $L_{g} / \operatorname{Re} d \leq 0.058$, the nonlinear damping force can be written as $F_{c}(t)=2 G F_{0} U \omega \cos \omega t$

$$
\begin{aligned}
& \left\{4 \omega^{2}\left[m_{2}\left(x_{3}{ }^{2}-\omega^{2}+y_{3}{ }^{2}\right)+x_{3}\left(m_{2}{ }^{2}-\omega^{2}+n_{2}{ }^{2}\right)\right]\right\} \cdot\left(-\omega^{2}\right)+ \\
& =\frac{2 G F_{0}}{x_{0}} \cdot \frac{\left\{2 \omega\left[\left(x_{3}{ }^{2}-\omega^{2}+y_{3}^{2}\right)\left(m_{2}^{2}-\omega^{2}+n_{2}^{2}\right)-4 m_{2} x_{3} \omega^{2}\right]\right\} \cdot\left(\frac{\omega A_{g}}{\rho L_{g}} R_{h}^{\prime}\right)}{\left\{4 \omega^{2}\left[m_{2}\left(x_{3}^{2}-\omega^{2}+y_{3}^{2}\right)+x_{3}\left(m_{2}^{2}-\omega^{2}+n_{2}^{2}\right)\right]\right\}^{2}+\left(-\omega^{2}\right)^{2}} v \\
& =\frac{2 G F_{0}}{x_{0}} \frac{\left\{4 \omega^{2}\left[m_{2}\left(x_{3}{ }^{2}-\omega^{2}+y_{3}^{2}\right)+x_{3}\left(m_{2}{ }^{2}-\omega^{2}+n_{2}{ }^{2}\right)\right]\right\} \cdot\left(-\omega^{2}\right)}{\left\{4 \omega^{2}\left[m_{2}\left(x_{3}{ }^{2}-\omega^{2}+y_{3}{ }^{2}\right)+x_{3}\left(m_{2}{ }^{2}-\omega^{2}+n_{2}{ }^{2}\right)\right]\right\}^{2}+\left(-\omega^{2}\right)^{2}} v \\
& +\frac{2 G F_{0}}{x_{0}} \cdot \frac{\left\{2 \omega\left[\left(x_{3}{ }^{2}-\omega^{2}+y_{3}^{2}\right)\left(m_{2}{ }^{2}-\omega^{2}+n_{2}^{2}\right)-4 m_{2} x_{3} \omega^{2}\right]\right\} \cdot\left(\frac{\omega A_{g}}{\rho L_{g}}\right)}{\left\{4 \omega^{2}\left[m_{2}\left(x_{3}{ }^{2}-\omega^{2}+y_{3}{ }^{2}\right)+x_{3}\left(m_{2}{ }^{2}-\omega^{2}+n_{2}{ }^{2}\right)\right]\right\}^{2}+\left(-\omega^{2}\right)^{2}} R_{h}^{\prime} v \\
& =\lambda_{9} v+\lambda_{10} R_{h}^{\prime} v=\lambda_{9} v+\lambda_{10} R_{h}\left(1+\tau_{1} v^{3 / 4}+\tau_{2} v\right) v \\
& \lambda_{9}=\frac{2 G F_{0}}{x_{0}} \frac{\left\{4 \omega^{2}\left[m_{2}\left(x_{3}^{2}-\omega^{2}+y_{3}^{2}\right)+x_{3}\left(m_{2}^{2}-\omega^{2}+n_{2}^{2}\right)\right]\right\} \cdot\left(-\omega^{2}\right)}{\left\{4 \omega^{2}\left[m_{2}\left(x_{3}^{2}-\omega^{2}+y_{3}^{2}\right)+x_{3}\left(m_{2}^{2}-\omega^{2}+n_{2}^{2}\right)\right]\right\}^{2}+\left(-\omega^{2}\right)^{2}} \\
& \lambda_{10}=\frac{2 G F_{0}}{x_{0}} \cdot \frac{\left\{2 \omega\left[\left(x_{3}^{2}-\omega^{2}+y_{3}^{2}\right)\left(m_{2}^{2}-\omega^{2}+n_{2}^{2}\right)-4 m_{2} x_{3} \omega^{2}\right]\right\} \cdot\left(\frac{\omega A_{g}}{\rho L_{g}}\right)}{\left\{4 \omega^{2}\left[m_{2}\left(x_{3}^{2}-\omega^{2}+y_{3}^{2}\right)+x_{3}\left(m_{2}^{2}-\omega^{2}+n_{2}^{2}\right)\right]\right\}^{2}+\left(-\omega^{2}\right)^{2}}
\end{aligned}
$$

The nonlinear elastic force can be written as 
$F_{k}(t)=-2 G F_{0} V \omega \sin \omega t$

$$
\begin{gathered}
\left\{4 \omega^{2}\left[m_{2}\left(x_{3}^{2}-\omega^{2}+y_{3}^{2}\right)+x_{3}\left(m_{2}^{2}-\omega^{2}+n_{2}^{2}\right)\right]\right\} \cdot\left(\frac{\omega A_{g}}{\rho L_{g}} R_{h}^{\prime}\right)- \\
=-\frac{2 G F_{0} \omega}{x_{0}} \cdot \frac{\left\{2 \omega\left[\left(x_{3}^{2}-\omega^{2}+y_{3}^{2}\right)\left(m_{2}^{2}-\omega^{2}+n_{2}^{2}\right)-4 m_{2} x_{3} \omega^{2}\right]\right\} \cdot\left(-\omega^{2}\right)}{\left\{4 \omega^{2}\left[m_{2}\left(x_{3}^{2}-\omega^{2}+y_{3}^{2}\right)+x_{3}\left(m_{2}^{2}-\omega^{2}+n_{2}^{2}\right)\right]\right\}^{2}+\left(-\omega^{2}\right)^{2}} x \\
=-\frac{2 G F_{0} \omega}{x_{0}} \cdot \frac{\left\{4 \omega^{2}\left[m_{2}\left(x_{3}^{2}-\omega^{2}+y_{3}^{2}\right)+x_{3}\left(m_{2}^{2}-\omega^{2}+n_{2}^{2}\right)\right]\right\} \cdot\left(\frac{\omega A_{g}}{\rho L_{g}}\right)}{\left\{4 \omega^{2}\left[m_{2}\left(x_{3}^{2}-\omega^{2}+y_{3}^{2}\right)+x_{3}\left(m_{2}^{2}-\omega^{2}+n_{2}^{2}\right)\right]\right\}^{2}+\left(-\omega^{2}\right)^{2}} R_{h}^{\prime} x \\
+\frac{2 G F_{0} \omega}{x_{0}} \cdot \frac{\left\{2 \omega\left[\left(x_{3}^{2}-\omega^{2}+y_{3}^{2}\right)\left(m_{2}^{2}-\omega^{2}+n_{2}^{2}\right)-4 m_{2} x_{3} \omega^{2}\right]\right\} \cdot\left(-\omega^{2}\right)}{\left\{4 \omega^{2}\left[m_{2}\left(x_{3}^{2}-\omega^{2}+y_{3}^{2}\right)+x_{3}\left(m_{2}^{2}-\omega^{2}+n_{2}^{2}\right)\right]\right\}^{2}+\left(-\omega^{2}\right)^{2} x} \\
=\lambda_{11} R_{h}^{\prime} x+\lambda_{12} x=\lambda_{11} R_{h}\left(1+\tau_{1} v^{3 / 4}+\tau_{2} v\right) x+\lambda_{12} x \\
\lambda_{11}=-\frac{2 G F_{0} \omega}{x_{0}} \cdot \frac{\left\{4 \omega^{2}\left[m_{2}\left(x_{3}^{2}-\omega^{2}+y_{3}^{2}\right)+x_{3}\left(m_{2}^{2}-\omega^{2}+n_{2}^{2}\right)\right]\right\} \cdot\left(\frac{\omega A_{g}}{\rho L_{g}}\right)}{\left\{4 \omega^{2}\left[m_{2}\left(x_{3}^{2}-\omega^{2}+y_{3}^{2}\right)+x_{3}\left(m_{2}^{2}-\omega^{2}+n_{2}^{2}\right)\right]\right\}^{2}+\left(-\omega^{2}\right)^{2}} \\
\lambda_{12}=\frac{2 G F_{0} \omega}{x_{0}} \cdot \frac{\left\{2 \omega\left[\left(x_{3}^{2}-\omega^{2}+y_{3}^{2}\right)\left(m_{2}^{2}-\omega^{2}+n_{2}^{2}\right)-4 m_{2} x_{3} \omega^{2}\right]\right\} \cdot\left(-\omega^{2}\right)}{\left\{4 \omega^{2}\left[m_{2}\left(x_{3}^{2}-\omega^{2}+y_{3}^{2}\right)+x_{3}\left(m_{2}^{2}-\omega^{2}+n_{2}^{2}\right)\right]\right\}^{2}+\left(-\omega^{2}\right)^{2}}
\end{gathered}
$$

When $L_{g} / \operatorname{Re} d>0.058$, damping force can be written as $F_{c}(t)=2 G F_{0} U \omega \cos \omega t$

$$
\begin{gathered}
\quad\left\{4 \omega^{2}\left[m_{2}\left(x_{3}^{2}-\omega^{2}+y_{3}^{2}\right)+x_{3}\left(m_{2}^{2}-\omega^{2}+n_{2}^{2}\right)\right]\right\} \cdot\left(-\omega^{2}\right)+ \\
=\frac{2 G F_{0}}{x_{0}} \cdot \frac{\left\{2 \omega\left[\left(x_{3}^{2}-\omega^{2}+y_{3}^{2}\right)\left(m_{2}^{2}-\omega^{2}+n_{2}^{2}\right)-4 m_{2} x_{3} \omega^{2}\right]\right\} \cdot\left(\frac{\omega A_{g}}{\rho L_{g}} R_{h}^{\prime}\right)}{\left\{4 \omega^{2}\left[m_{2}\left(x_{3}^{2}-\omega^{2}+y_{3}^{2}\right)+x_{3}\left(m_{2}^{2}-\omega^{2}+n_{2}^{2}\right)\right]\right\}^{2}+\left(-\omega^{2}\right)^{2}} v \\
=\frac{2 G F_{0}}{x_{0}} \cdot \frac{\left\{4 \omega^{2}\left[m_{2}\left(x_{3}^{2}-\omega^{2}+y_{3}^{2}\right)+x_{3}\left(m_{2}^{2}-\omega^{2}+n_{2}^{2}\right)\right]\right\} \cdot\left(-\omega^{2}\right)}{\left\{4 \omega^{2}\left[m_{2}\left(x_{3}^{2}-\omega^{2}+y_{3}^{2}\right)+x_{3}\left(m_{2}^{2}-\omega^{2}+n_{2}^{2}\right)\right]\right\}^{2}+\left(-\omega^{2}\right)^{2}} v \\
+\frac{2 G F_{0}}{x_{0}} \cdot \frac{\left\{2 \omega\left[\left(x_{3}^{2}-\omega^{2}+y_{3}^{2}\right)\left(m_{2}^{2}-\omega^{2}+n_{2}^{2}\right)-4 m_{2} x_{3} \omega^{2}\right]\right\} \cdot\left(\frac{\omega A_{g}}{\rho L_{g}}\right)}{\left\{4 \omega^{2}\left[m_{2}\left(x_{3}^{2}-\omega^{2}+y_{3}^{2}\right)+x_{3}\left(m_{2}^{2}-\omega^{2}+n_{2}^{2}\right)\right]\right\}^{2}+\left(-\omega^{2}\right)^{2}} R_{h}^{\prime} v
\end{gathered}
$$




$$
\begin{gathered}
=\lambda_{13} v+\lambda_{14} R_{h}^{\prime} v=\lambda_{13} v+\lambda_{14} R_{h}\left(1+\tau_{3} v\right) v \\
\lambda_{13}=\frac{2 G F_{0}}{x_{0}} \cdot \frac{\left\{4 \omega^{2}\left[m_{2}\left(x_{3}^{2}-\omega^{2}+y_{3}^{2}\right)+x_{3}\left(m_{2}^{2}-\omega^{2}+n_{2}^{2}\right)\right]\right\} \cdot\left(-\omega^{2}\right)}{\left\{4 \omega^{2}\left[m_{2}\left(x_{3}^{2}-\omega^{2}+y_{3}^{2}\right)+x_{3}\left(m_{2}{ }^{2}-\omega^{2}+n_{2}{ }^{2}\right)\right]\right\}^{2}+\left(-\omega^{2}\right)^{2}} \\
\lambda_{14}=\frac{2 G F_{0}}{x_{0}} \cdot \frac{\left\{2 \omega\left[\left(x_{3}{ }^{2}-\omega^{2}+y_{3}{ }^{2}\right)\left(m_{2}{ }^{2}-\omega^{2}+n_{2}{ }^{2}\right)-4 m_{2} x_{3} \omega^{2}\right]\right\} \cdot\left(\frac{\omega A_{g}}{\rho L_{g}}\right)}{\left\{4 \omega^{2}\left[m_{2}\left(x_{3}{ }^{2}-\omega^{2}+y_{3}{ }^{2}\right)+x_{3}\left(m_{2}{ }^{2}-\omega^{2}+n_{2}{ }^{2}\right)\right]\right\}^{2}+\left(-\omega^{2}\right)^{2}}
\end{gathered}
$$

Elastic force can be written as

$$
\begin{aligned}
& F_{k}(t)=-2 G F_{0} V \omega \sin \omega t \\
& =\frac{-\frac{2 G F_{0} \omega}{x_{0}} \cdot\left\{4 \omega^{2}\left[m_{2}\left(x_{3}^{2}-\omega^{2}+y_{3}^{2}\right)+x_{3}\left(m_{2}^{2}-\omega^{2}+n_{2}^{2}\right)\right]\right\} \cdot\left(\frac{\omega A_{g}}{\rho L_{g}}\right)}{\left\{4 \omega^{2}\left[m_{2}\left(x_{3}^{2}-\omega^{2}+y_{3}^{2}\right)+x_{3}\left(m_{2}^{2}-\omega^{2}+n_{2}^{2}\right)\right]\right\}^{2}+\left(-\omega^{2}\right)^{2}} R_{h}^{\prime} x+ \\
& \frac{\frac{2 G F_{0} \omega}{x_{0}} \cdot\left\{2 \omega\left[\left(x_{3}^{2}-\omega^{2}+y_{3}^{2}\right)\left(m_{2}^{2}-\omega^{2}+n_{2}^{2}\right)-4 m_{2} x_{3} \omega^{2}\right]\right\} \cdot\left(-\omega^{2}\right)}{\left\{4 \omega^{2}\left[m_{2}\left(x_{3}^{2}-\omega^{2}+y_{3}^{2}\right)+x_{3}\left(m_{2}^{2}-\omega^{2}+n_{2}^{2}\right)\right]\right\}^{2}+\left(-\omega^{2}\right)^{2}} x \\
& =\lambda_{15} R_{h}\left(1+\tau_{3} v\right) x+\lambda_{16} x \\
& \lambda_{15}=\frac{-\frac{2 G F_{0} \omega}{x_{0}} \cdot\left\{4 \omega^{2}\left[m_{2}\left(x_{3}{ }^{2}-\omega^{2}+y_{3}{ }^{2}\right)+x_{3}\left(m_{2}{ }^{2}-\omega^{2}+n_{2}{ }^{2}\right)\right]\right\} \cdot\left(\frac{\omega A_{g}}{\rho L_{g}}\right)}{\left\{4 \omega^{2}\left[m_{2}\left(x_{3}^{2}-\omega^{2}+y_{3}^{2}\right)+x_{3}\left(m_{2}^{2}-\omega^{2}+n_{2}{ }^{2}\right)\right]\right\}^{2}+\left(-\omega^{2}\right)^{2}} \\
& \lambda_{16}=\frac{\frac{2 G F_{0} \omega}{x_{0}} \cdot\left\{2 \omega\left[\left(x_{3}{ }^{2}-\omega^{2}+y_{3}{ }^{2}\right)\left(m_{2}{ }^{2}-\omega^{2}+n_{2}{ }^{2}\right)-4 m_{2} x_{3} \omega^{2}\right]\right\} \cdot\left(-\omega^{2}\right)}{\left\{4 \omega^{2}\left[m_{2}\left(x_{3}{ }^{2}-\omega^{2}+y_{3}{ }^{2}\right)+x_{3}\left(m_{2}{ }^{2}-\omega^{2}+n_{2}{ }^{2}\right)\right]\right\}^{2}+\left(-\omega^{2}\right)^{2}}
\end{aligned}
$$

Similarly, the displacement of the damping piston can be calculated by the same method. The discussion is still divided into two cases.

1) Equation (15) has a pair of unequal real roots and a pair of conjugate complex roots. Displacement of the damping piston can be expressed as

$$
\begin{gathered}
x(t)=H\left\{2 U \cos \omega t-2 V \sin \omega t+c_{1} e^{x_{1} t}+c_{2} e^{x_{2} t}+2 e^{m_{1} t}\left(X \cos n_{1} t-Y \sin n_{1} t\right)\right\} \\
D_{1}=-2 n_{1}^{2}\left\{\left(m_{1}^{2}-n_{1}^{2}+\omega^{2}\right)\left(2 m_{1}-x_{1}-x_{2}\right)+2 m_{1}\left[\left(m_{1}-x_{1}\right)\left(m_{1}-x_{2}\right)-n_{1}^{2}\right]\right\} \\
D_{2}=2 n_{1}\left\{\left(m_{1}^{2}-n_{1}^{2}+\omega^{2}\right)\left[\left(m_{1}-x_{1}\right)\left(m_{1}-x_{2}\right)-n_{1}^{2}\right]-2 m_{1} n_{1}^{2}\left(2 m_{1}-x_{1}-x_{2}\right)\right\}
\end{gathered}
$$




$$
\begin{aligned}
& D_{3}=m_{1}^{2}-n_{1}^{2}+A_{1} m_{1}+A_{5}, D_{4}=2 m_{1} n_{1}+A_{1} n_{1} \\
& X=\left(D_{1} D_{3}+D_{2} D_{4}\right) /\left(D_{1}^{2}+D_{2}^{2}\right), Y=\left(D_{1} D_{4}-D_{2} D_{3}\right) /\left(D_{1}^{2}+D_{2}^{2}\right) \\
& c_{1}=\frac{x_{1}^{2}+A_{1} x_{1}+A_{5}}{\left(x_{1}-x_{2}\right)\left(x_{1}^{2}+\omega^{2}\right)\left[\left(x_{1}-m_{1}\right)^{2}+n_{1}^{2}\right]} \\
& c_{2}=\frac{x_{2}^{2}+A_{1} x_{2}+A_{5}}{\left(x_{2}-x_{1}\right)\left(x_{2}^{2}+\omega^{2}\right)\left[\left(x_{2}-m_{1}\right)^{2}+n_{1}^{2}\right]}
\end{aligned}
$$

2) Equation (15) has two pairs of conjugate complex roots. Displacement of the damping piston can be expressed as

$$
x(t)=H\left\{\begin{array}{l}
2 U \cos \omega t-2 V \sin \omega t+2 e^{x_{3} t}\left(M \cos y_{3} t-N \sin y_{3} t\right)+ \\
2 e^{m_{2} t}\left(X \cos n_{2} t-Y \sin n_{2} t\right)
\end{array}\right\}
$$

$$
\begin{aligned}
& H=F_{0} \omega / M, B_{1}=4 \omega^{2}\left[m_{2}\left(x_{3}^{2}-\omega^{2}+y_{3}^{2}\right)+x_{3}\left(m_{2}^{2}-\omega^{2}+n_{2}^{2}\right)\right] \\
& B_{2}=2 \omega\left[\left(x_{3}^{2}-\omega^{2}+y_{3}^{2}\right)\left(m_{2}^{2}-\omega^{2}+n_{2}^{2}\right)-4 m_{2} x_{3} \omega^{2}\right] \\
& B_{3}=A_{5}-\omega^{2}, B_{4}=A_{1} \omega, U=\left(B_{1} B_{3}+B_{2} B_{4}\right) /\left(B_{1}^{2}+B_{2}^{2}\right), V=\left(B_{1} B_{4}-B_{2} B_{3}\right) /\left(B_{1}^{2}+B_{2}^{2}\right) \\
& D_{1}=-4 y_{3}^{2}\left\{\left(x_{3}-m_{2}\right)\left(x_{3}^{2}+\omega^{2}-y_{3}^{2}\right)+x_{3}\left[\left(x_{3}-m_{2}\right)^{2}-y_{3}^{2}+n_{2}^{2}\right]\right\} \\
& D_{2}=2 y_{3}\left\{\left(x_{3}^{2}+\omega^{2}-y_{3}^{2}\right)\left[\left(x_{3}-m_{2}\right)^{2}-y_{3}^{2}+n_{2}^{2}\right]-4 x_{3} y_{3}^{2}\left(x_{3}-m_{2}\right)\right\} \\
& D_{3}=x_{3}^{2}-y_{3}^{2}+A_{1} x_{3}+A_{5}, D_{4}=y_{3} A_{1}+2 x_{3} y_{3} \\
& M=\left(D_{1} D_{3}+D_{2} D_{4}\right) /\left(D_{1}^{2}+D_{2}^{2}\right), N=\left(D_{1} D_{4}-D_{2} D_{3}\right) /\left(D_{1}^{2}+D_{2}^{2}\right) \\
& E_{1}=-4 n_{2}^{2}\left\{\left(m_{2}-x_{3}\right)\left(m_{2}^{2}-n_{2}^{2}+\omega\right)+m_{2}\left[\left(m_{2}-x_{3}\right)^{2}-n_{2}^{2}+y_{3}^{2}\right]\right\} \\
& E_{2}=2 n_{2}\left\{\left(m_{2}^{2}-n_{2}^{2}+\omega^{2}\right)\left[\left(m_{2}-x_{3}\right)^{2}-n_{2}^{2}+y_{3}^{2}\right]-4 m_{2} n_{2}^{2}\left(m_{2}-x_{3}\right)\right\} \\
& E_{3}=m_{2}^{2}-n_{2}^{2}+A_{1} m_{2}+A_{5} \\
& E_{4}=n_{2} A_{1}+2 m_{2} n_{2} \\
& X=\left(E_{1} E_{3}+E_{2} E_{4}\right) /\left(E_{1}^{2}+E_{2}^{2}\right), Y=\left(E_{1} E_{4}-E_{2} E_{3}\right) /\left(E_{1}^{2}+E_{2}^{2}\right)
\end{aligned}
$$

\subsection{Model validation}

To verify the theoretical model established in the previous section, the CFD model is established by using the finite element software ADINA. According to the discussion in Section 1.1, the damper can be simplified by a single tube fluid viscous damper and two springs connected to the damping piston. The fluid model is shown in Fig.2. The structure model is shown in Fig.3, in which the spring element in ADINA is used on 
both sides of the damping piston. The stiffness of the spring element is $2.4 \times 10^{4} \mathrm{~N} / \mathrm{m}$, which is equal to the stiffness of the bellows in Fig.1. The principal stiffness of the damper is $4.8 \times 10^{4} \mathrm{~N} / \mathrm{m}$. To save calculation time, a $1 / 4$ model is used. Symmetric boundary conditions are used in the model to meet the flow conditions. After the calculation, when the pressure differential force is extracted, it needs to be multiplied by 4 to obtain the final pressure differential force. The simulation parameters of the model are shown in Table 1. The damping fluid is silicone oil. In the calculation process, we assumed that the viscosity of the silicone oil remains constant. Usually, the temperature of silicone oil will rise after the damper working for a long time, but the viscosity will decrease after the temperature rises, which will lead to the reduction of energy consumption and the temperature of silicone oil will further decrease. In addition, the damper itself will dissipate heat to the external environment, so it is a dynamic balance process. Therefore, the influence of temperature on viscosity is not considered in this paper. In the model, the external excitation force is sinusoidal and the amplitude is $48 \mathrm{~N}$.

Table 1 Simulation parameters

\begin{tabular}{cc}
\hline Parameter & Value \\
\hline Diameter $\mathrm{d} / \mathrm{mm}$ & 2 \\
Length $1 / \mathrm{mm}$ & 30 \\
Stiffness of spring k/(N/m) & $2.4 \times 104 \mathrm{~N} / \mathrm{m}$ \\
Viscosity $v / \mathrm{cst}$ & 100 \\
Bulk modulus $\beta / \mathrm{MPa}$ & 100 \\
Density $\rho /(\mathrm{Kg} / \mathrm{m} 3)$ & 903 \\
Mass of damping piston M/ Kg & 1 \\
Diameter of chamber D/mm & 31.5 \\
Length of chamber $\mathrm{L} / \mathrm{mm}$ & 50 \\
Frequency f/Hz & $10 / 50 / 100 / 200$ \\
Amplitude of force $\mathrm{F} / \mathrm{N}$ & 48 \\
\hline
\end{tabular}

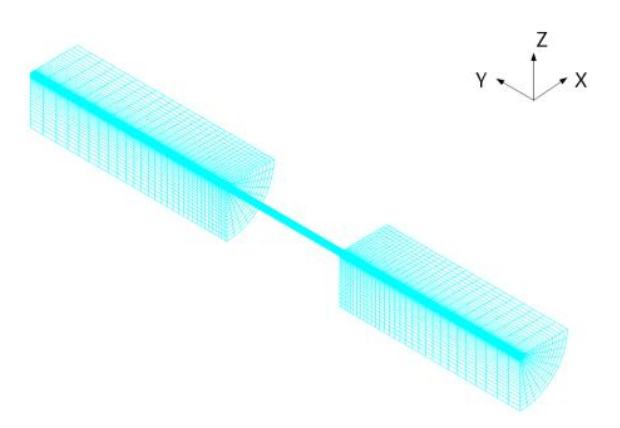

Fig.2 Mesh of fluid

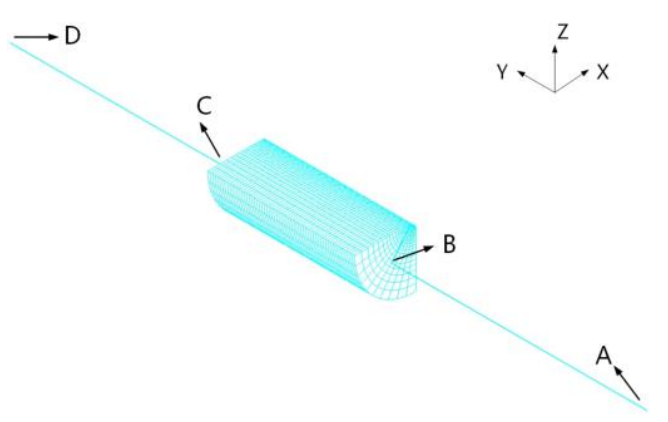

Fig.3 Mesh of structure 
Fig.4 is the comparison of the pressure differential force at different frequencies when the viscosity is 100 cst. Fig.4 (a), (b), (c), and (d) correspond to the pressure differential forces at frequencies of $10 \mathrm{~Hz}, 50 \mathrm{~Hz}, 100 \mathrm{~Hz}$, and $200 \mathrm{~Hz}$. It can be seen that the results of the theoretical model are consistent with those of the CFD model. Fig.5 is the comparison of displacement of damping piston at different frequencies when the viscosity is 100 cst. Fig.5 (a), (b), (c), and (d) correspond to the displacement of the damping piston at $10 \mathrm{~Hz}, 50 \mathrm{~Hz}, 100 \mathrm{~Hz}$, and $200 \mathrm{~Hz}$. It can be seen that the results of the theoretical model are consistent with those calculated by the CFD model. Table 2 is the comparison between the amplitude of pressure differential force and displacement, the theoretical model and CFD model are consistent. However, when the frequency is $50 \mathrm{~Hz}$, the results have some differences, the reason is being analyzed at present.
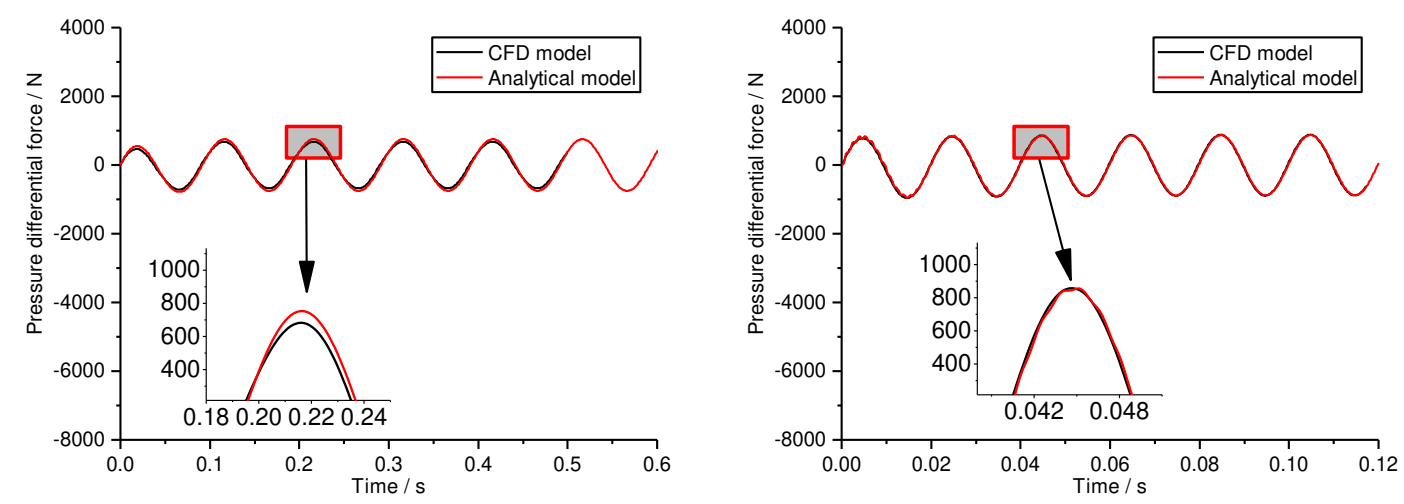

(a) $\mathrm{f}=10 \mathrm{~Hz}$
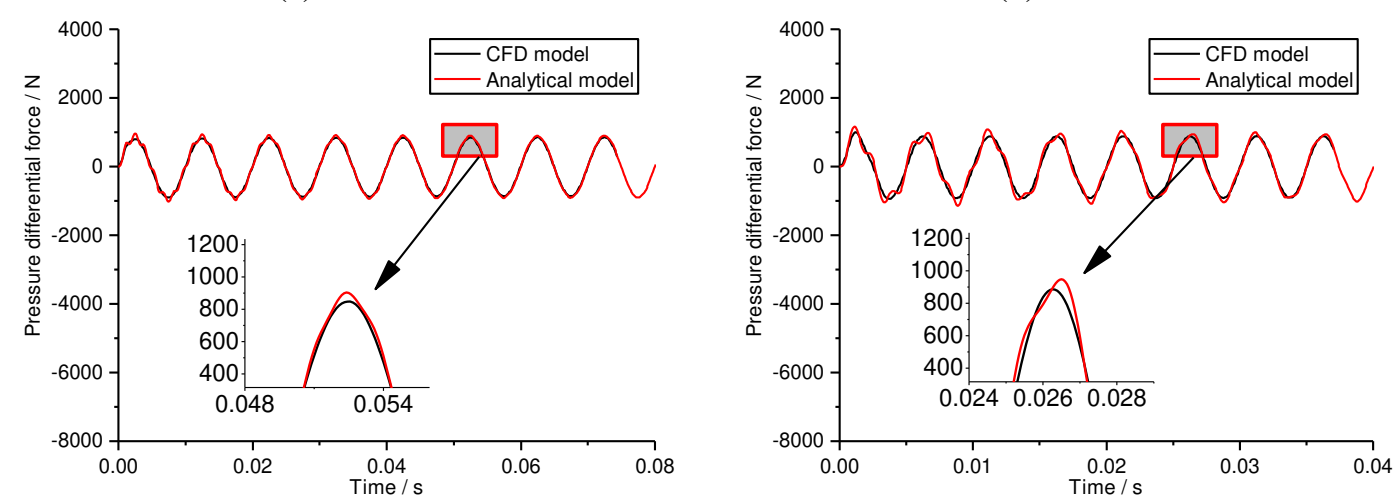

(c) $\mathrm{f}=100 \mathrm{~Hz}$

(d) $\mathrm{f}=200 \mathrm{~Hz}$

Fig.4 Comparison of pressure differential forces at different frequencies when viscosity is $100 \mathrm{cst}$ 


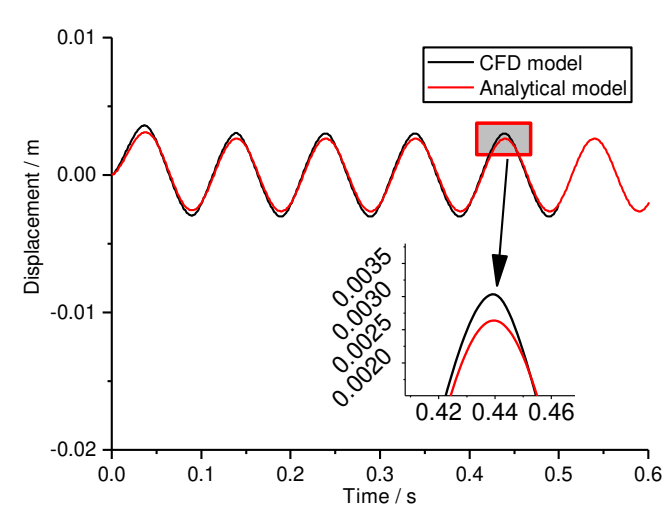

(a) $\mathrm{f}=10 \mathrm{~Hz}$

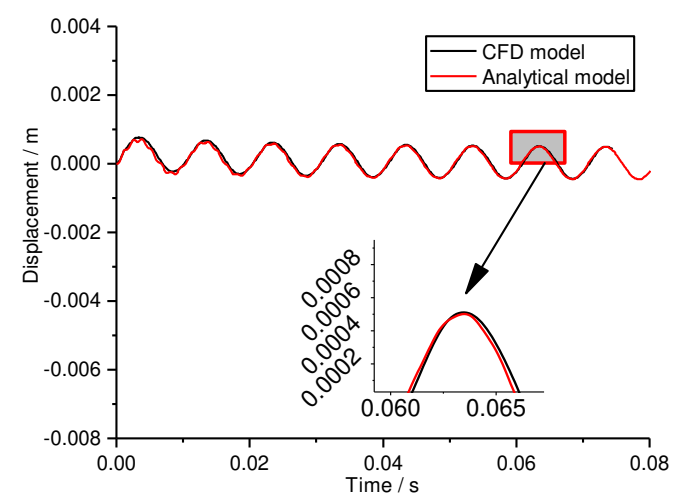

(c) $\mathrm{f}=100 \mathrm{~Hz}$

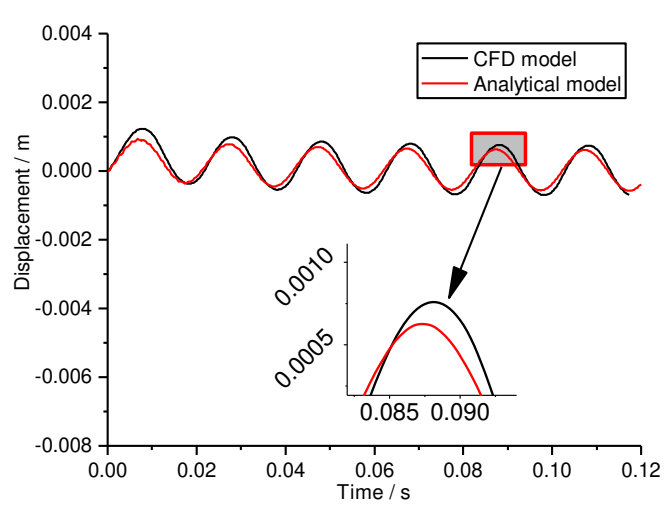

(b) $\mathrm{f}=50 \mathrm{~Hz}$

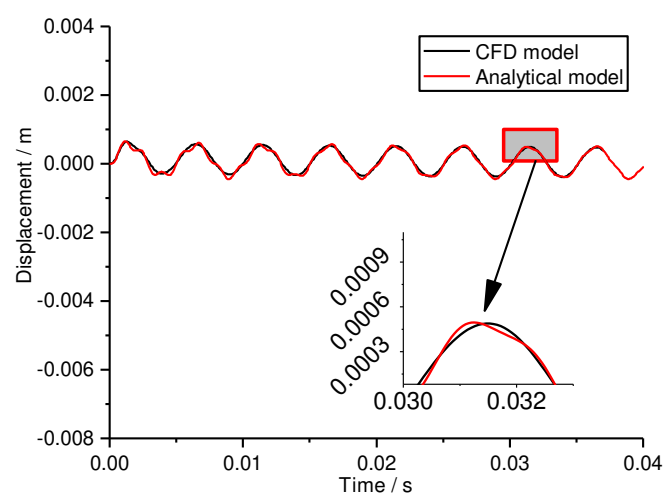

(d) $\mathrm{f}=200 \mathrm{~Hz}$

Fig.5 Comparison of displacement of damping piston at different frequencies when viscosity is 100

cst

Table 2 Comparison of amplitude of pressure differential force and displacement

\begin{tabular}{ccccc}
\hline & $\begin{array}{c}\text { Frequency / } \\
\mathrm{Hz}\end{array}$ & CFD Model & $\begin{array}{c}\text { Theoretical } \\
\text { Model }\end{array}$ & Relative error \\
\hline \multirow{2}{*}{ Pressure } & $10 \mathrm{~Hz}$ & 683 & 753 & $10.2 \%$ \\
differential & $50 \mathrm{~Hz}$ & 880 & 877 & $0.3 \%$ \\
force $/ \mathrm{N}$ & $100 \mathrm{~Hz}$ & 848 & 896 & $5.7 \%$ \\
& $200 \mathrm{~Hz}$ & 889 & 950 & $6.9 \%$ \\
\hline & $10 \mathrm{~Hz}$ & 3.03 & 2.64 & $12.9 \%$ \\
Displacement & $50 \mathrm{~Hz}$ & 0.74 & 0.59 & $20.3 \%$ \\
of damping & $100 \mathrm{~Hz}$ & 0.51 & 0.47 & $7.8 \%$ \\
piston /mm & $200 \mathrm{~Hz}$ & 0.5 & 0.45 & $10 \%$ \\
\hline
\end{tabular}

\section{Nonlinear characteristics analysis}

\subsection{Analysis of hydraulic resistance correction coefficient}

As has been mentioned, the hydraulic resistance correction coefficient can influence the nonlinear component in pressure differential force. In this section, the 
hydraulic resistance correction coefficients at different damping orifice diameters, lengths, and viscosities are analyzed. Simulation parameters are shown in Table 3.

Table 3 Simulation parameters

\begin{tabular}{ccc}
\hline Cases & Parameters & Value \\
\hline \multirow{2}{*}{ Case 1 } & Damping orifice diameter/ mm & $1 / 1.5 / 2 / 2.5 / 3 / 3.5 / 4 / 4.5 / 5$ \\
& Damping orifice length/ mm & 30 \\
Viscosity/ cst & 100 \\
\hline \multirow{2}{*}{ Case 2 } & Damping orifice diameter/ mm & 5 \\
& Damping orifice length/ mm & $30 / 35 / 40 / 45 / 50 / 55 / 60$ \\
Case 3 & Viscosity/ cst & 100 \\
\hline \multirow{2}{*}{ Camping orifice diameter/ mm } & 5 \\
& Damping orifice length/ mm & 30 \\
\hline
\end{tabular}

The outer diameter of the damper $D$ is $31.5 \mathrm{~mm}$. The bulk modulus of silicone oil $\beta$ is $100 \mathrm{MPa}$. Silicone oil density $\rho$ is $903 \mathrm{Kg} / \mathrm{m}^{3}$. The amplitude of external force $F$ is $48 \mathrm{~N}$.

Fig. 6 shows the displacement of the damping piston at different damping orifice diameters. It can be seen from the figure that there is a resonance peak in the displacement curve. When the damping orifice diameter is $5 \mathrm{~mm}$, the resonance peak occurs at $7 \mathrm{~Hz}$, and the amplitude of damping piston displacement is $0.00416 \mathrm{~m}$, which is much larger than the static displacement of the damping piston. As the damping orifice diameter decreases, the resonance peak decreases gradually. When the damping orifice diameter decreases smaller than $3 \mathrm{~mm}$, the frequency of resonance peak increases obviously. When the damping orifice diameter decreases to $1 \mathrm{~mm}$, there is a high-order resonance peak at $100 \mathrm{~Hz}$, and the displacement of the damping piston is $7.81 \times 10^{-5} \mathrm{~m}$. Fig.7 shows the hydraulic resistance correction coefficient at different damping orifice diameters. If the hydraulic resistance is not corrected, the coefficient of hydraulic resistance should be 1 . The nonlinear term of velocity is included in the correction coefficient, which leads to nonlinear stiffness and damping. The larger the correction coefficient is, the more obvious the nonlinearity is. Corresponding to Fig.6, when the damping orifice diameter is $5 \mathrm{~mm}$, the correction coefficient of hydraulic resistance increases significantly at the same resonance frequency, and the maximum value is 2.83 , which indicates that the nonlinearity of the damper will be significant at $7 \mathrm{~Hz}$. The reason is that when the damping piston resonates, it moves very fast, which will accelerate the flow speed in the damping orifice, resulting in a larger nonlinear term of velocity. With the decrease of damping orifice diameter, the correction 
coefficient at resonance frequency decreases gradually, but it increases at a higher-order resonance peak. When the damping orifice diameter is $1 \mathrm{~mm}$ and the frequency is 90 $\mathrm{Hz}$, the correction coefficient is 1.52 , which means the damper still has nonlinearity. In general, when the diameter of the damping orifice changes, the correction coefficient is only greater than 1 near the resonance peak but close to 1 at most other frequencies. This is mainly due to the fast movement speed of the damping piston near the resonance peak. However, at higher frequencies, the correction coefficient is smaller because the movement amplitude of the damping piston is very small and the movement speed is relatively small. That means that for the three-parameter fluid viscous damper considering the principal stiffness, the nonlinearity of the damper will be significant near the resonance peak.

Fig. 8 shows the displacement of the damping piston at different damping orifice lengths. Similarly, it can be seen from the figure that the displacement curve has an obvious first-order resonance peak and second-order resonance peak. The first resonance peak occurs near $7 \mathrm{~Hz}$. When the damping orifice length is $30 \mathrm{~mm}$, the displacement of the damping piston can reach $4.16 \times 10^{-3} \mathrm{~m}$. When the damping orifice length increases to $60 \mathrm{~mm}$, this displacement can reach $2.27 \times 10^{-3} \mathrm{~m}$. The second resonance peak occurs in the range of $237 \mathrm{~Hz}$ to $279 \mathrm{~Hz}$. At the second resonance peak, the maximum displacement of the damping piston is only $2.12 \times 10^{-6} \mathrm{~m}$. In the frequency band between the first and second resonance peaks, the displacement is nearly the same. Fig.9 shows the hydraulic resistance correction factor at different orifice lengths. In Fig.9, the correction coefficient curve also has a resonance peak at 7 $\mathrm{Hz}$. When the damping orifice length is $30 \mathrm{~mm}$, the correction coefficient can reach 2.83, which indicates the damper has significant nonlinearity. When the damping orifice length is $60 \mathrm{~mm}$, the correction coefficient is only 1.53 . The reason is that the larger the displacement resonance peak is, the faster the velocity of the fluid in the damping orifice will be, resulting in the larger nonlinear term of velocity and the larger correction coefficient. However, the increase of the correction coefficient is not obvious at the high-order resonance peak. This is mainly due to the displacement amplitude of the high-order resonance peak is only $10^{-6} \mathrm{~m}$, and the fluid velocity in the damping orifice is very small. This also indicates that the nonlinearity of the damper is obvious if the resonance peak is obvious. 
Fig.10 shows displacement of the damping piston at different viscosities. It can be seen from the figure that there is a significant first-order formant in the displacement curve, but the higher-order resonance peak is not obvious. Besides, the displacement curve is nearly the same above $20 \mathrm{~Hz}$. When the viscosity is $100 \mathrm{cst}$, the displacement at the resonance peak is $4.16 \times 10^{-3} \mathrm{~m}$. When the viscosity is $1000 \mathrm{cst}$, there is no obvious resonance peak in the displacement curve, which indicates that the damper has a significant suppression on the resonance peak. Fig.11 shows the hydraulic resistance correction coefficient at different viscosities. It can be seen that the correction coefficient reaches the maximum value at the resonance frequency. When the viscosity is $100 \mathrm{cst}$, the correction coefficient is 2.83 , because the correction coefficient determines the strength of nonlinearity, it means that the nonlinearity at resonance peak will be significant. When the viscosity is $1000 \mathrm{cst}$, the correction coefficient is only 1.022. When the frequency is greater than $30 \mathrm{~Hz}$, there exists a high-order resonance peak, but not obvious, so the correction coefficient is still close to 1 at most frequencies beyond $30 \mathrm{~Hz}$, which indicates the nonlinearity is not obvious at these frequencies.

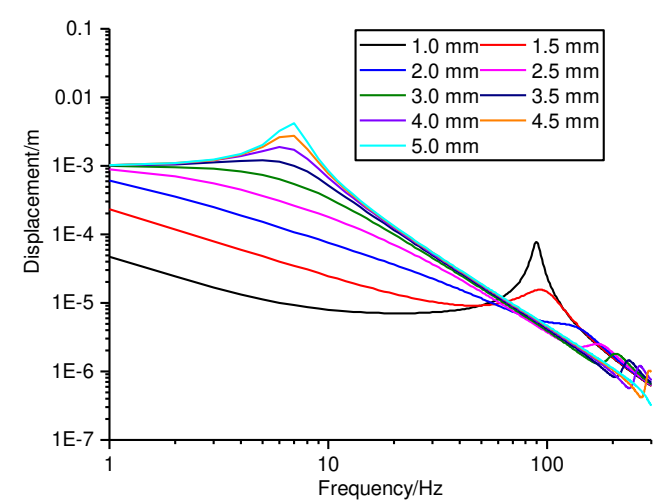

Fig.6 Displacement of damping piston at different damping orifice diameters

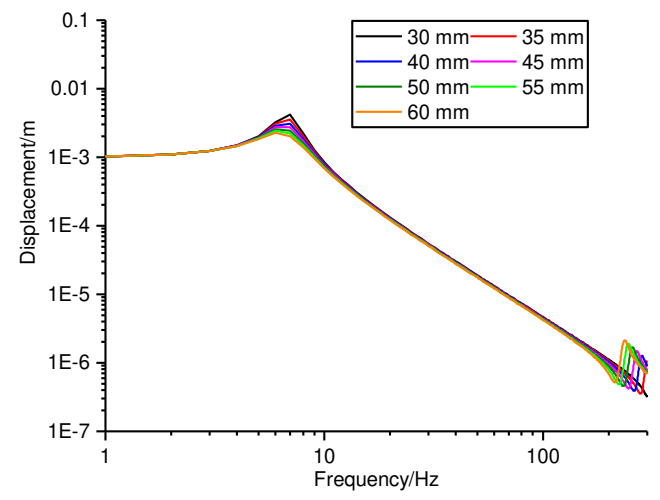

Fig.8 Displacement of damping piston at different damping orifice lengths

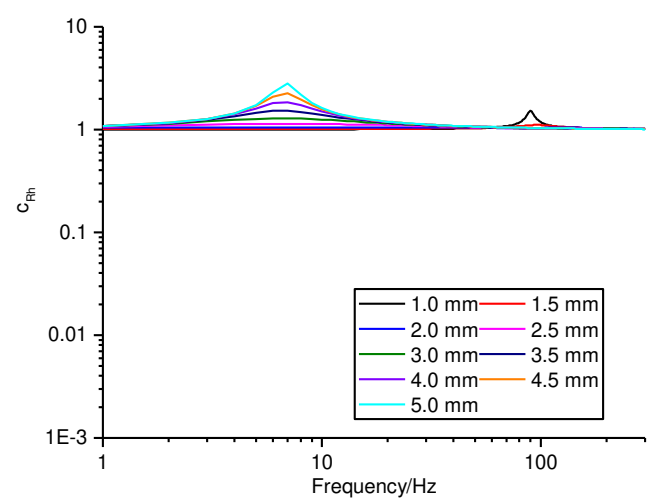

Fig.7 Hydraulic resistance correction factor at different damping orifice diameters

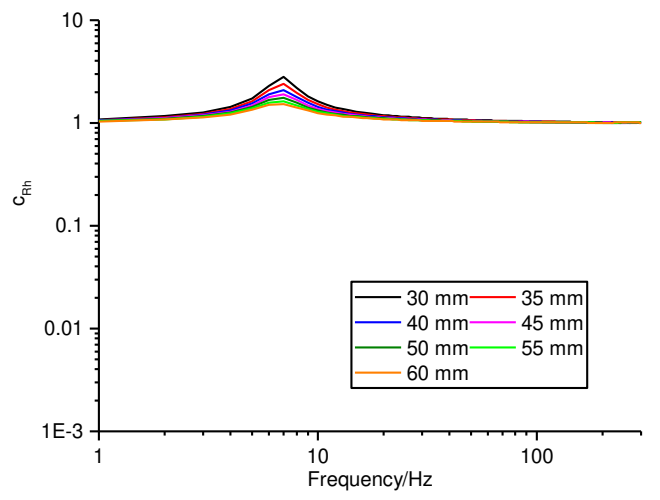

Fig.9 Hydraulic resistance correction factor at different damping orifice lengths 


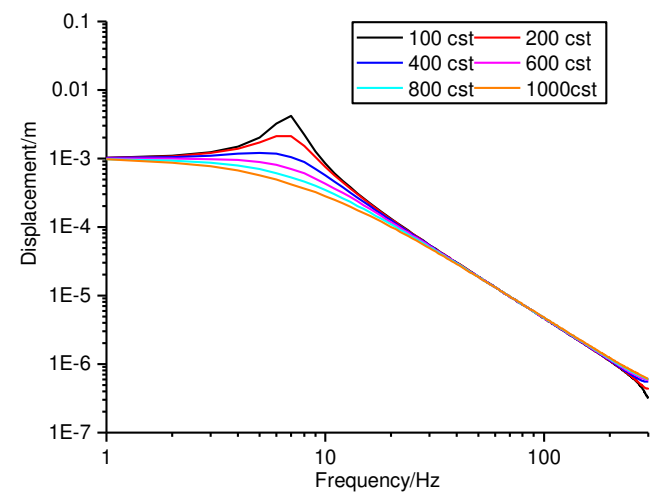

Fig.10 Displacement of damping piston at different viscosities

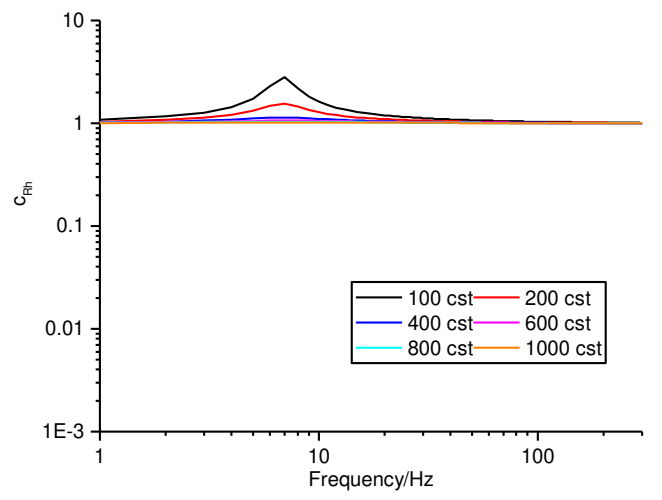

Fig.11 Hydraulic resistance correction factor at different viscosities

\subsection{Nonlinear characteristics of pressure differential force and hysteresis loop}

In this section, nonlinear characteristics of the pressure differential force and hysteresis loop at the resonance peak will be analyzed. Table 4 shows the frequencies and amplitudes of the first-order and the second-order resonance peak at different damping orifice diameters. Since there is no first-order resonance peak when the damping orifice diameter is $5 \mathrm{~mm}$, the second-order resonance frequency and amplitude are not given and are represented by "None" in the table. Table 5 shows the frequencies and amplitudes of the first-order and the second-order resonance peak at different damping orifice lengths. Table 6 shows the frequencies and amplitudes of the first-order and the second-order resonance peak at different viscosities. Since there are no secondorder resonance peaks when damping orifice lengths are $30 \mathrm{~mm}$ and $35 \mathrm{~mm}$, the secondorder resonance frequencies and amplitudes are not given, and are represented by "None". Fig.12 shows the pressure differential force at the first-order resonance peak. If the uncorrected hydraulic resistance is used, the curve of pressure differential force should be sine or cosine curve. It can be seen from the enlarged diagram that the pressure difference force curve exists local distortion, which indicates there exists a nonlinear component in pressure differential force. The nonlinearity of the pressure differential force tends to decrease with the decrease of the damping orifice diameter. Fig.13 shows the pressure differential force at the second-order resonance peak. It can 
be seen that the pressure differential force is a complete sine curve, and there is no local distortion, which indicates the nonlinearity of the damper at the second-order resonance peak is not obvious. The reason is that the damper has huge hydraulic stiffness in the high-frequency domain, which will hinder the movement of the damping piston, and resulting in a significant decrease of damping piston displacement. From Table 4, it can be seen that the displacement of the second-order resonance peak is only um, so the nonlinearity of the damper is not obvious at this time. Fig.14 shows the pressure differential force at the first-order resonance peak for different damping orifice lengths. It can be seen from the local enlarged diagram that the curve exists distortion, indicating that there exist nonlinear components at this time. However, with the increase of orifice length, the nonlinearity of the damper decreases. Fig. 15 shows the pressure differential force at the second-order resonance peak for the different orifice lengths. It can be seen from the figure that the curve is a complete sine curve, which indicates there is no nonlinear component in pressure differential force at this time. Fig.16 shows the pressure differential force at the first resonance peak for different viscosities. It can be seen from the local enlarged diagram that the curve is not a complete sine curve at this time, indicating that there is also a nonlinear component in pressure differential force.

Table 4 Resonance frequencies and amplitudes of the first-order and second-order resonance peak at

\begin{tabular}{|c|c|c|c|}
\hline Diameter $/ \mathrm{mm}$ & & 1st resonance peak & 2nd resonance peak \\
\hline 3.5 & $\begin{array}{l}\text { Frequency } / \mathrm{Hz} \\
\text { Amplitude } / \mathrm{m}\end{array}$ & $\begin{array}{c}5.0 \\
1.217 \times 10-3\end{array}$ & $\begin{array}{c}236.5 \\
1.449 \times 10-6\end{array}$ \\
\hline 4.0 & $\begin{array}{l}\text { Frequency } / \mathrm{Hz} \\
\text { Amplitude /m }\end{array}$ & $\begin{array}{c}6.2 \\
1.884 \times 10-3\end{array}$ & $\begin{array}{c}265.5 \\
1.208 \times 10-6\end{array}$ \\
\hline 4.5 & $\begin{array}{l}\text { Frequency } / \mathrm{Hz} \\
\text { Amplitude /m }\end{array}$ & $\begin{array}{c}6.6 \\
2.911 \times 10-3 \\
\end{array}$ & $\begin{array}{c}294.2 \\
1.031 \times 10-6\end{array}$ \\
\hline 5.0 & $\begin{array}{l}\text { Frequency } / \mathrm{Hz} \\
\text { Amplitude } / \mathrm{m}\end{array}$ & $\begin{array}{c}6.8 \\
4.353 \times 10-3\end{array}$ & $\begin{array}{l}\text { None } \\
\text { None }\end{array}$ \\
\hline
\end{tabular}

Table 5 Resonance frequencies and amplitudes $\mathrm{f}$ the first-order and second-order resonance peak at different damping orifice lengths

\begin{tabular}{cccc}
\hline Length $/ \mathrm{mm}$ & & $1^{\text {st }}$ resonance peak & $2^{\text {nd }}$ resonance peak \\
\hline \multirow{2}{*}{30} & Frequency $/ \mathrm{Hz}$ & 6.8 & None \\
& Amplitude $/ \mathrm{m}$ & $4.353 \times 10^{-3}$ & None \\
\hline \multirow{2}{*}{35} & Frequency $/ \mathrm{Hz}$ & 6.7 & None \\
& Amplitude $/ \mathrm{m}$ & $3.769 \times 10^{-3}$ & None \\
\hline \multirow{2}{*}{40} & Frequency $/ \mathrm{Hz}$ & 6.6 & 283.2
\end{tabular}




\begin{tabular}{cccc} 
& Amplitude $/ \mathrm{m}$ & $3.327 \times 10^{-3}$ & $1.277 \times 10^{-6}$ \\
\hline \multirow{2}{*}{45} & Frequency /Hz & 6.5 & 268.5 \\
& Amplitude $/ \mathrm{m}$ & $2.983 \times 10^{-3}$ & $1.479 \times 10^{-6}$ \\
\hline \multirow{2}{*}{50} & Frequency /Hz & 6.4 & 256.2 \\
& Amplitude /m & $2.709 \times 10^{-3}$ & $1.688 \times 10^{-6}$ \\
\hline \multirow{2}{*}{55} & Frequency /Hz & 6.4 & 245.6 \\
& Amplitude $/ \mathrm{m}$ & $2.485 \times 10^{-3}$ & $1.906 \times 10^{-6}$ \\
\hline \multirow{2}{*}{60} & Frequency /Hz & 6.3 & 236.5 \\
& Amplitude $/ \mathrm{m}$ & $2.301 \times 10^{-3}$ & $2.126 \times 10^{-6}$ \\
\hline
\end{tabular}

Table 6 Resonance frequencies and amplitudes $\mathrm{f}$ the first-order and second-order resonance peak at different viscosities

\begin{tabular}{cccc}
\hline Viscosity /cst & & 1st resonance peak & 2nd resonance peak \\
\hline \multirow{2}{*}{100} & Frequency $/ \mathrm{Hz}$ & 6.8 & None \\
& Amplitude $/ \mathrm{m}$ & $4.353 \times 10-3$ & None \\
\hline \multirow{2}{*}{200} & Frequency $/ \mathrm{Hz}$ & 6.5 & None \\
& Amplitude $/ \mathrm{m}$ & $2.225 \times 10-3$ & None \\
\hline \multirow{2}{*}{400} & Frequency $/ \mathrm{Hz}$ & 5.2 & None \\
& Amplitude $/ \mathrm{m}$ & $1.219 \times 10-3$ & None \\
\hline
\end{tabular}

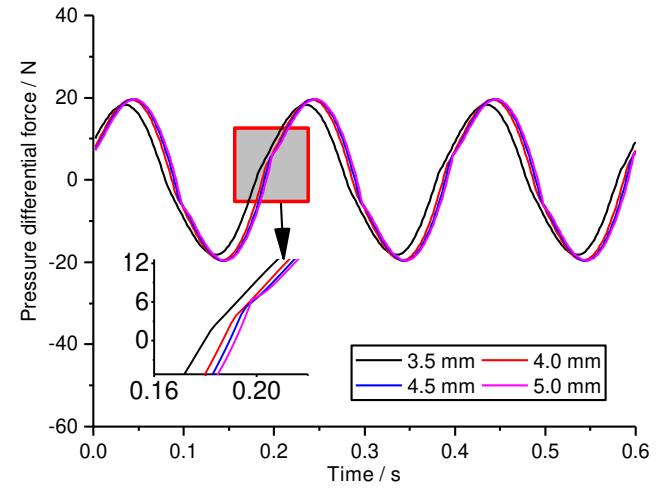

Fig.12 Pressure differential force of the firstorder resonance peak at different damping orifice diameters

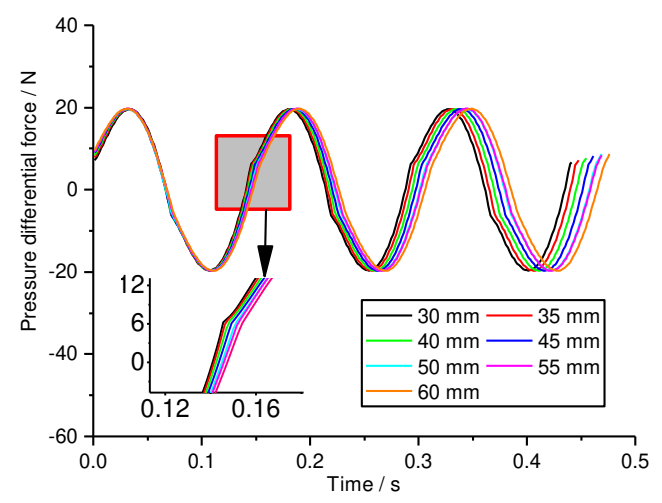

Fig.14 Pressure differential force of the firstorder resonance peak at different damping orifice lengths

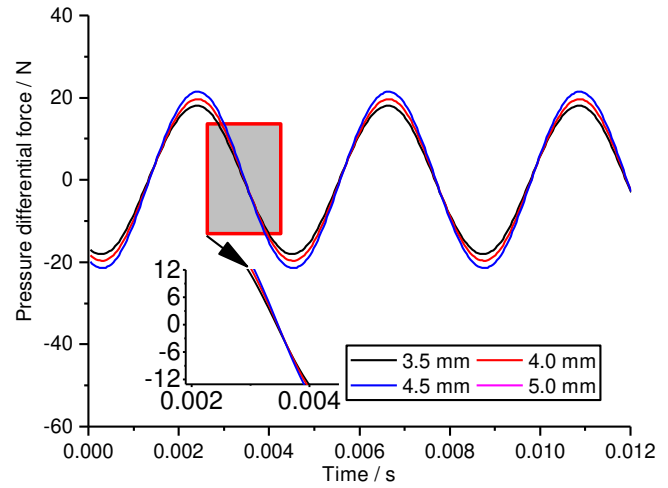

Fig.13 Pressure differential force of the second-order resonance peak at different damping orifices

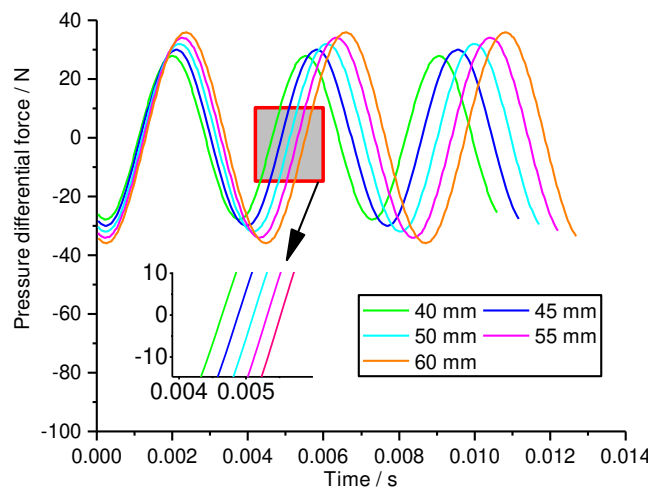

Fig.15 Pressure differential force of the second-order resonance peak at different damping orifice lengths 


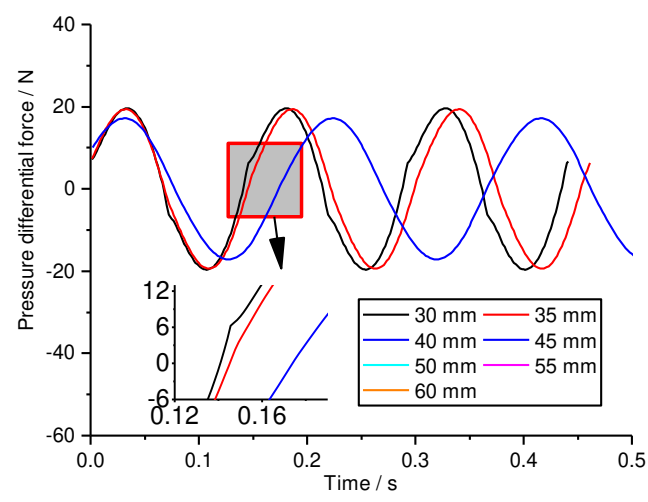

Fig.16 Pressure differential force of the first-order resonance peak at different viscosities

Fig.17 shows the hysteresis loop of the first-order resonance peak at different damping orifice diameters. Since the corrected hydraulic resistance contains the nonlinear term of velocity, the model using the corrected hydraulic resistance is the nonlinear model. To study the nonlinear characteristics, we compare it with the linear model. It can be seen from the figure that the hysteresis loop has some differences between the nonlinear model and linear model. Because the linear model does not contain the nonlinear term of velocity, the hysteresis loop is a standard ellipse. However, the hysteresis loop of the nonlinear model exists local distortion. The inclination of the ellipse is caused by the hydraulic stiffness of the damper.

Fig.18 shows the hysteresis loop at the second-order resonance peak. It can be seen that the hysteresis loops of the two models are nearly the same, which is different from the hysteresis loop at the first-order resonance peak. That means the nonlinearity at the second-order resonance peak is not obvious. The main reason is that at the second-order resonance peak, the displacement amplitude of damping piston is only um level, the velocity of the fluid in the damping orifice will be smaller, which leads to a small nonlinear term of velocity in the corrected hydraulic resistance, so the nonlinearity of the damper is not obvious.

Table 7 shows the energy consumption of the damper at the first-order and secondorder resonance peaks. It can be seen from the table that the energy consumption calculated by the nonlinear model at the first resonant peak is slightly larger than that calculated by the linear model, which indicates that the nonlinear characteristics can 
help to enhance the energy consumption of the damper at the resonance peak. However, at the second-order resonant peak, the energy consumption calculated by the nonlinear model is slightly smaller than that calculated by the linear model.

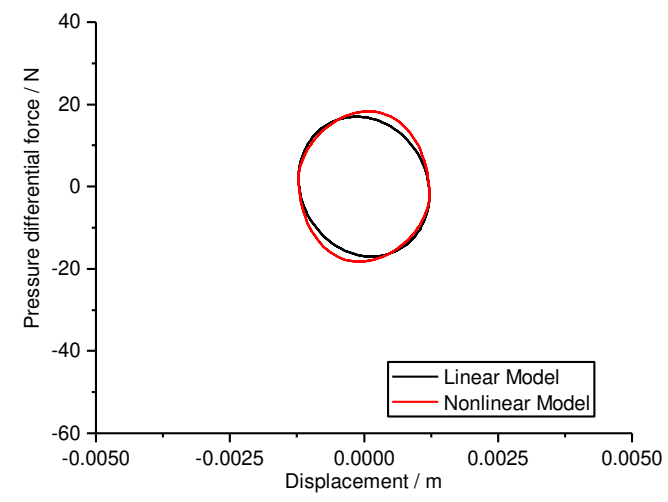

a) $\mathrm{d}=3.5 \mathrm{~mm}$

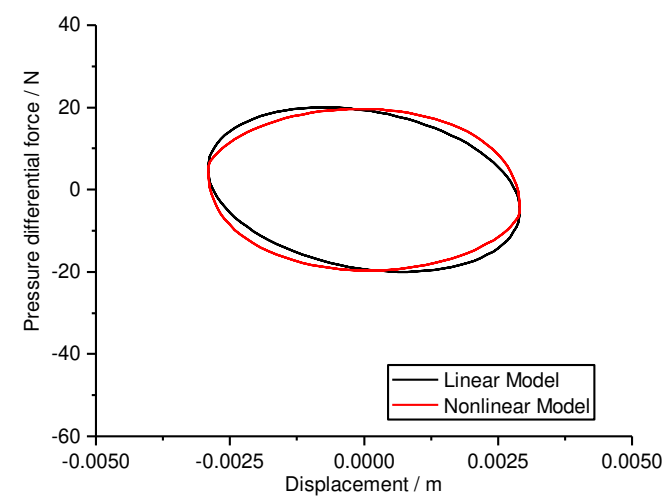

c) $\mathrm{d}=4.5 \mathrm{~mm}$

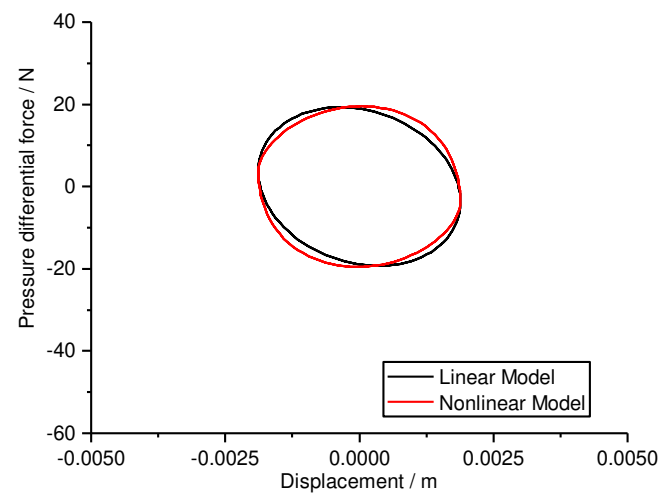

b) $\mathrm{d}=4.0 \mathrm{~mm}$

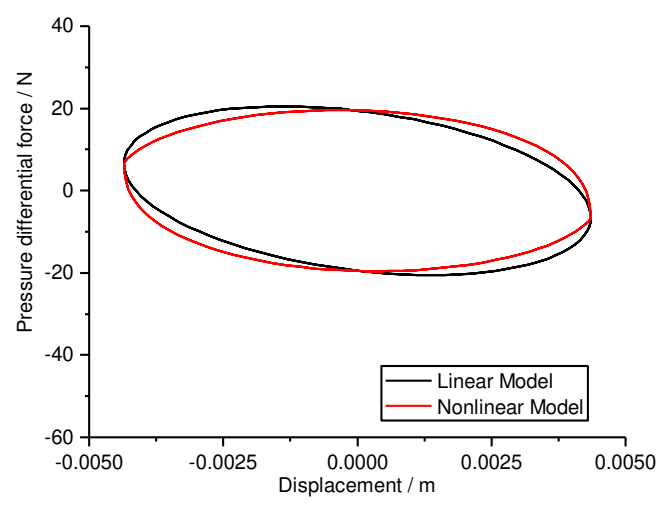

d) $\mathrm{d}=5.0 \mathrm{~mm}$

Fig. 17 Hysteresis loop of at the first-order resonance peak

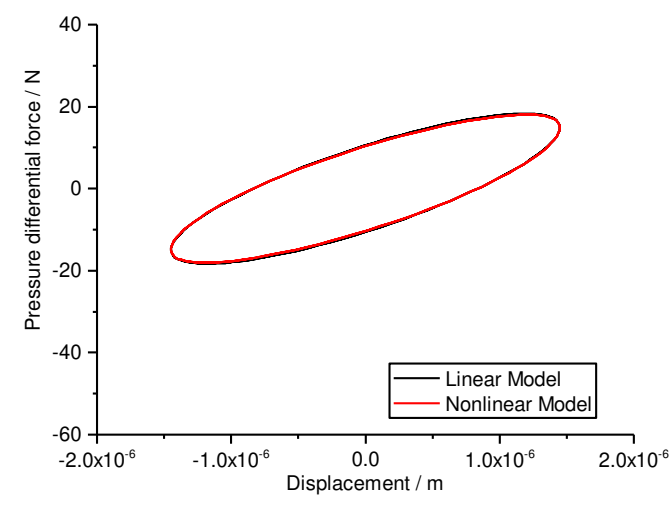

a) $\mathrm{d}=3.5 \mathrm{~mm}$

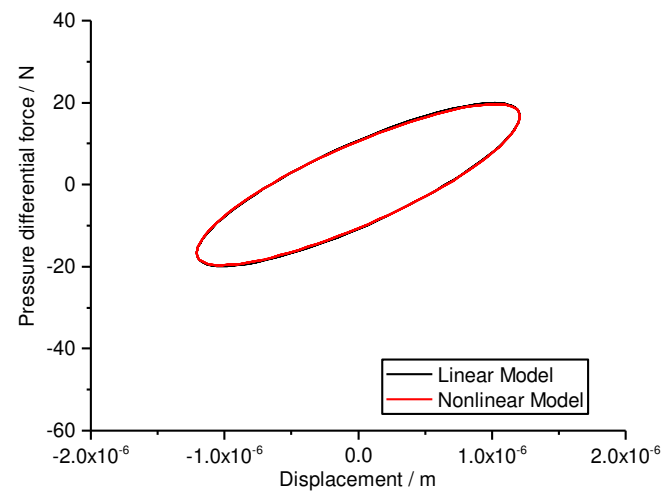

b) $\mathrm{d}=4.0 \mathrm{~mm}$ 


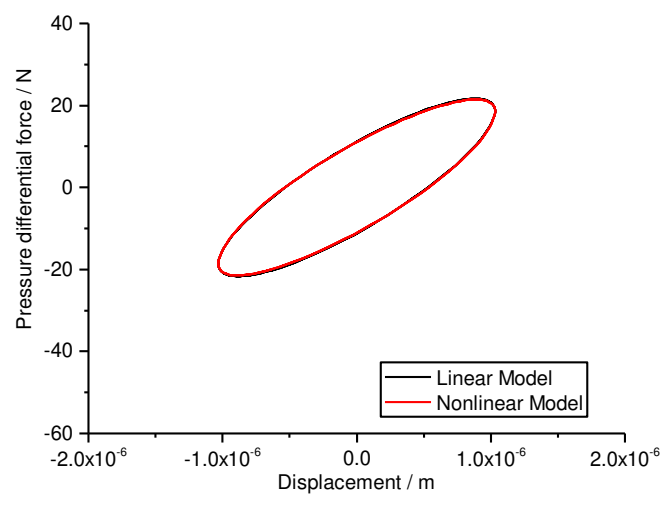

c) $\mathrm{d}=4.5 \mathrm{~mm}$

Fig.18 Hysteresis loop of at the second-order resonance peak

Table 7 Energy consumption of the first-order and second-order resonance peak at different damping

$$
\text { orifice }
$$

\begin{tabular}{ccccc}
\hline & \multicolumn{2}{c}{$\begin{array}{c}\text { Energy consumption at the first-order } \\
\text { Diameter } / \mathrm{mm}\end{array}$} & \multicolumn{2}{c}{$\begin{array}{c}\text { Energy consumption at the second- } \\
\text { order resonance peak } / \mathrm{J}\end{array}$} \\
& Linear model & Nonlinear model & Linear model & Nonlinear model \\
\hline 3.5 & 0.1931 & 0.2069 & $1.4279 \times 10-4$ & $1.4021 \times 10-4$ \\
4.0 & 0.3355 & 0.3458 & $1.2150 \times 10-4$ & $1.1998 \times 10-4$ \\
4.5 & 0.5302 & 0.5385 & $1.0739 \times 10-4$ & $1.0642 \times 10-4$ \\
5.0 & 0.7979 & 0.8017 & None & None \\
\hline
\end{tabular}

\subsection{Energy consumption}

The energy consumption of the damper can reflect the performance of suppressing the resonance peak. In this section, the energy consumption of the damper is analyzed at different damping orifice diameters, lengths, and viscosities. The simulation parameters are shown in Table 8 . The damping energy consumptions calculated by the linear model and nonlinear model are compared.

Fig.19 shows the energy consumption at different damping orifice diameters. It can be seen from the figure that the results have some differences between the linear model and the nonlinear model at the resonance peak. The energy consumption calculated by the nonlinear model is larger than that calculated by the linear model. That means nonlinear characteristics can increase the energy consumption of the damper near the resonance peak, which is beneficial to the suppression of the resonance 
peak. In the high-frequency domain, the energy consumption of these two models is nearly the same. This is mainly due to the small displacement amplitude of the damping piston in the high-frequency domain, only um level. This will lead to the small velocity of the fluid in the damping orifice, therefore the nonlinear velocity term in hydraulic resistance will be small. At this time, it can be concluded that the nonlinear damping is almost equal to the linear damping, which means that in the high-frequency domain, the nonlinear model and linear model have no obvious difference. According to the results of the four cases in Fig.19, the amplitude of resonance peak increases obviously with the increase of the damping orifice diameter, but the energy consumption of the damper calculated by the nonlinear model is still greater than that calculated by the linear model.

Fig.20 shows the comparison of energy consumption at different damping orifice lengths. When damping orifice length is $20 \mathrm{~mm}$ and $40 \mathrm{~mm}$, energy consumption calculated by the nonlinear model near the resonance peak is greater than that of the linear model. However, when damping orifice lengths are $60 \mathrm{~mm}$ and $80 \mathrm{~mm}$, energy consumption calculated by the nonlinear model is near the same as the linear model. This is mainly because when the resonance peak is large, the movement of the damping piston will be strong, and the nonlinear velocity term in the hydraulic resistance can not be ignored, more vibration energy will be consumed by nonlinear damping. However, the reason is opposite when has a small resonance peak. Besides, it can be seen from Fig.20 that when the orifice lengths are $60 \mathrm{~mm}$ and $80 \mathrm{~mm}$, the second-order resonance peak appears, but the energy consumptions of the nonlinear model and linear model are nearly the same at the second resonance peak. It mainly because the displacement amplitude of the damping piston is very small at the second-order resonance peak, which leads to a small nonlinear velocity term in the hydraulic resistance, it makes the nonlinear model and linear model tend to be consistent.

Table 8 Simulation parameters

Cases 


\begin{tabular}{ccc}
\hline \multirow{3}{*}{ Case 1 } & Damping orifice diameter/mm & $1 / 5 / 8 / 10$ \\
& Damping orifice length/mm & 30 \\
& Viscosity/ cst & 100 \\
\hline \multirow{3}{*}{ Case 2 } & Damping orifice diameter/mm & 5 \\
& Damping orifice length/ mm & $20 / 40 / 60 / 80$ \\
& Viscosity/ cst & 100 \\
\hline \multirow{2}{*}{ Case 3 } & Damping orifice diameter/mm & 5 \\
& Damping orifice length/ mm & 20 \\
& Viscosity/ cst & $200 / 400 / 600 / 1000$ \\
\hline
\end{tabular}

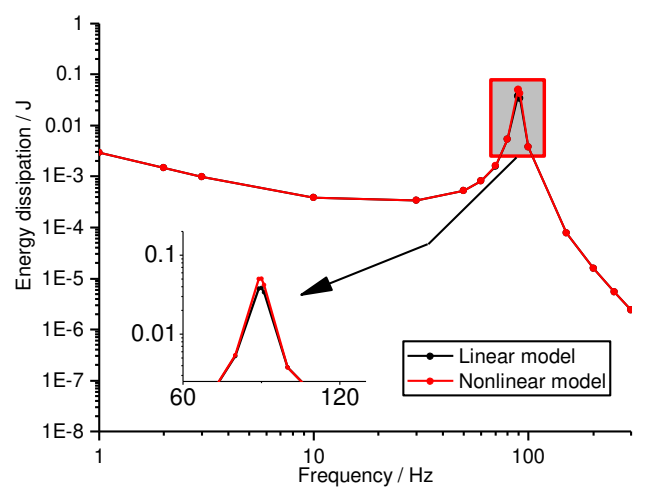

a) $\mathrm{d}=1.0 \mathrm{~mm}$

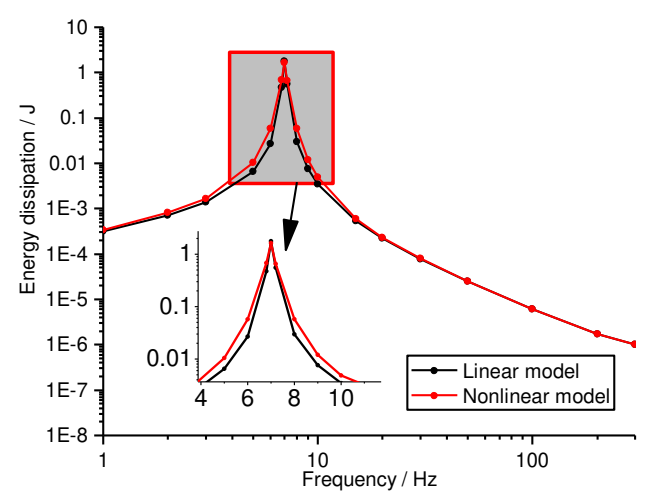

c) $\mathrm{d}=8.0 \mathrm{~mm}$

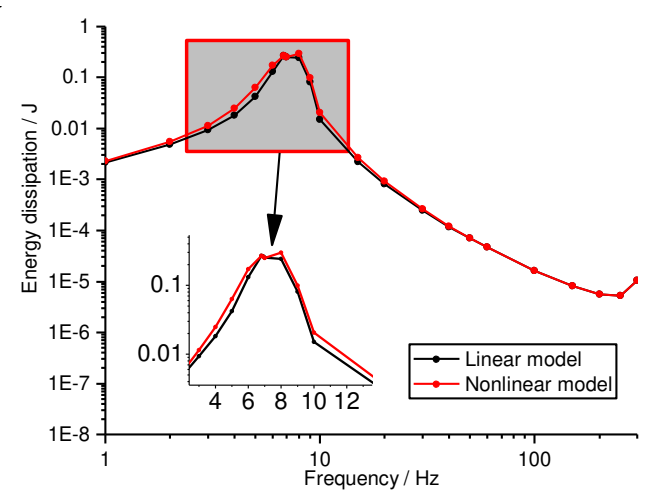

b) $\mathrm{d}=5.0 \mathrm{~mm}$

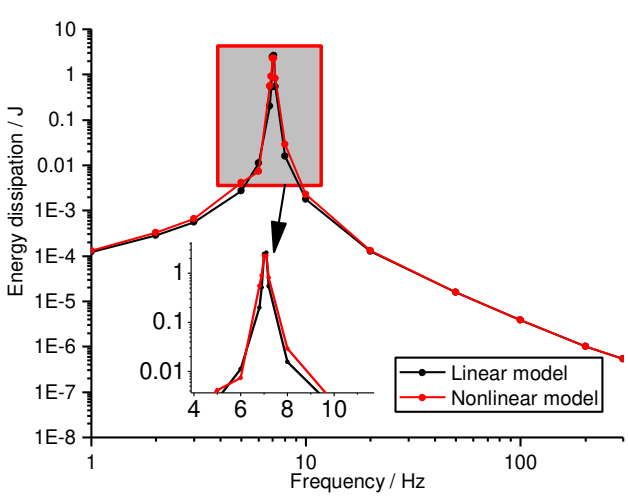

d) $\mathrm{d}=10.0 \mathrm{~mm}$

Fig.19 Energy consumption at different damping orifice diameters

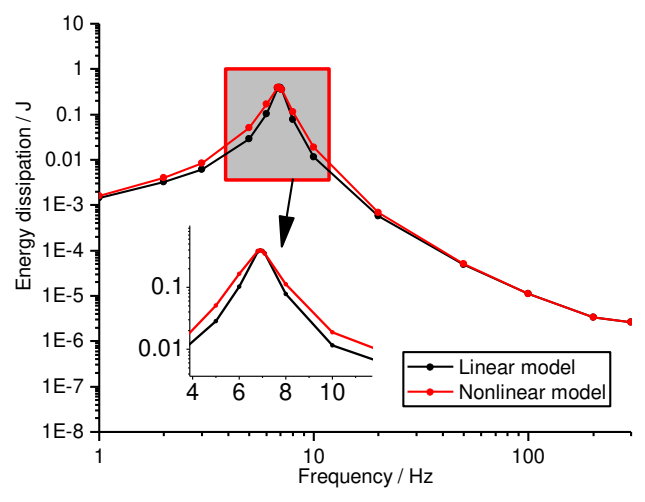

a) $\mathrm{Lg}=20 \mathrm{~mm}$

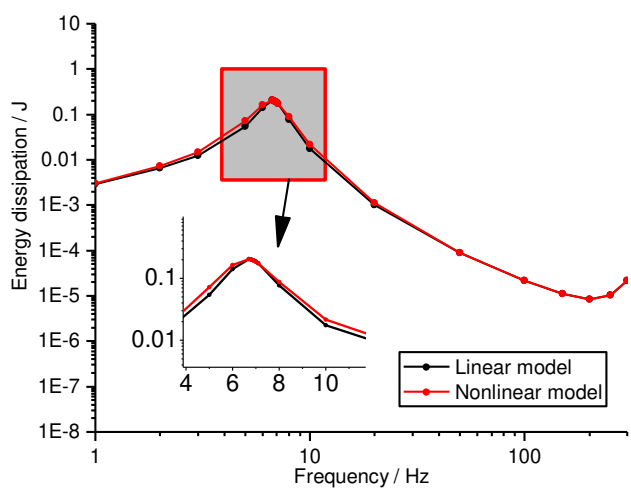

b) $\mathrm{Lg}=40 \mathrm{~mm}$ 


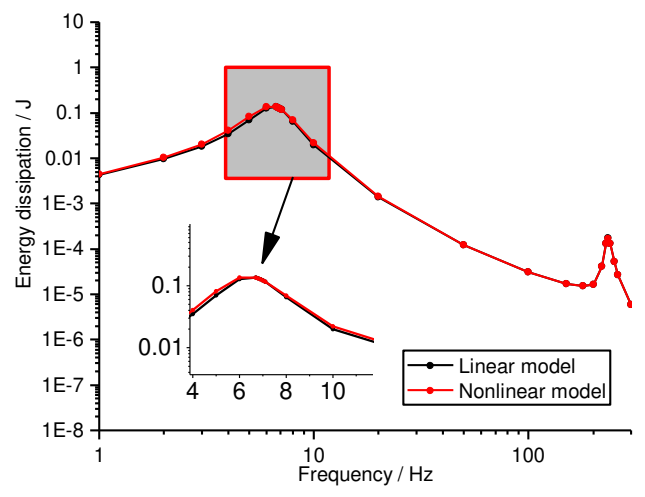

c) $\mathrm{Lg}=60 \mathrm{~mm}$

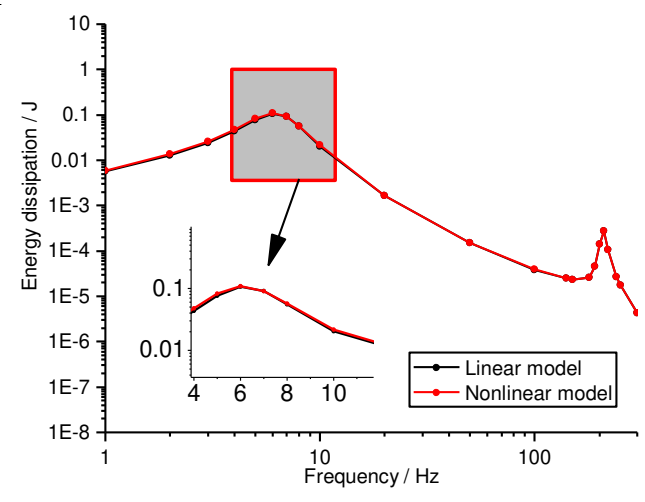

d) $\mathrm{Lg}=80 \mathrm{~mm}$

Fig.20 Energy consumption at different damping orifice lengths

Fig.21 shows the comparison of energy consumptions at different viscosities. It can be seen that when the viscosity is 200 cst, the damping energy consumption calculated by the nonlinear model is greater than that calculated by the linear model. But in the high-frequency domain, the energy consumption of nonlinear damping is slightly larger than linear damping. When viscosities are 200 cst, 600 cst, and 1000 cst, the energy consumption of nonlinear damping and linear damping is nearly the same both at the resonance peak and high-frequency domain. It can be concluded that the energy consumption of nonlinear damping at the first-order resonance peak is larger than that of linear damping at a low viscosity. This is mainly because of the high flow velocity in the damping orifice at low viscosity, which leads to large nonlinear velocity terms and nonlinear damping. When the viscosity is high, the velocity of the fluid in the damping orifice is relatively slow, which leads to a small nonlinear velocity term. At this time, the energy consumption of nonlinear damping is nearly the same as linear damping.

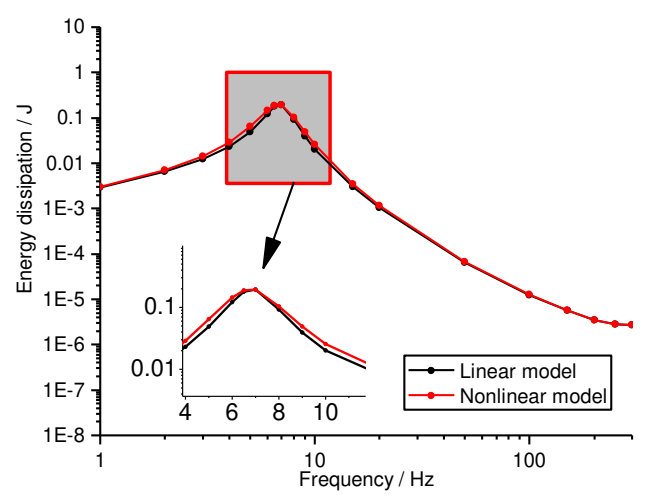

a) $v=200 \mathrm{cst}$

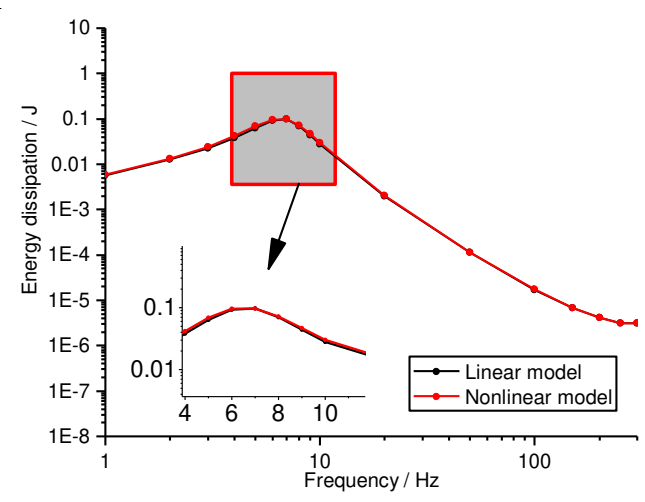

b) $\mathrm{v}=400 \mathrm{cst}$ 


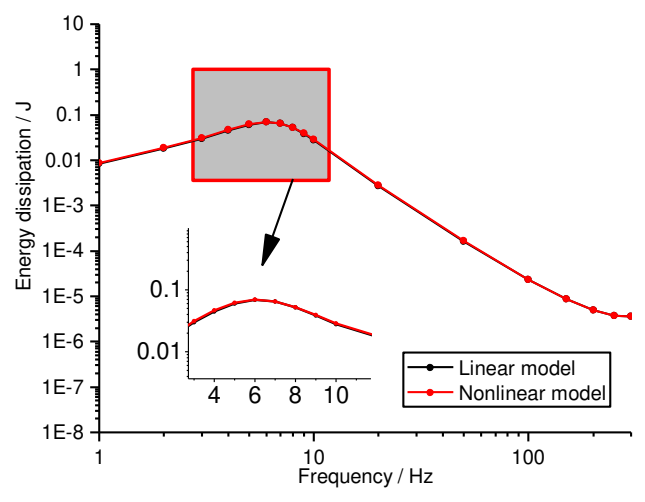

c) $v=600 \mathrm{cst}$

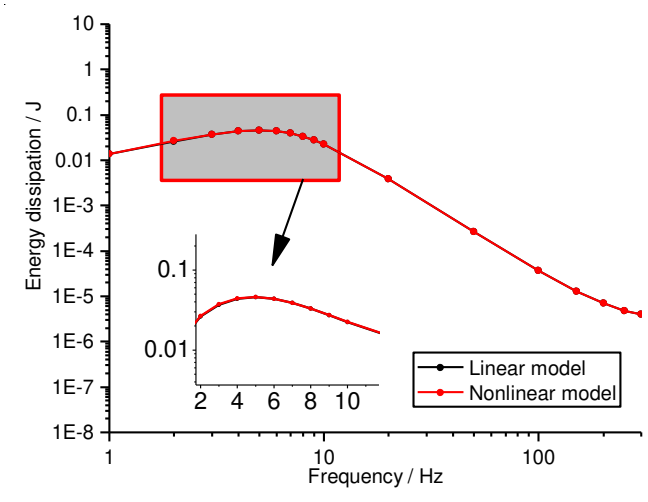

d) $\mathrm{v}=1000 \mathrm{cst}$

Fig.21 Energy consumption at different damping orifice viscosities

\section{Conclusion}

In this paper, a nonlinear model of a three-parameter fluid viscous damper considering the principal stiffness and hydraulic stiffness is proposed. Based on this model, the displacement response and the hydraulic resistance correction coefficient of the damper are analyzed. Besides, the energy consumption of nonlinear damping and linear damping at different damping orifice diameters, lengths, and viscosities are compared. The results show that there exist first-order and second-order resonance peaks when considering the principal stiffness and hydraulic stiffness. Near the resonance peak, the correction coefficient of hydraulic resistance is greater than 1 , while in the high-frequency domain, the correction coefficient of hydraulic resistance is almost 1. This means that the nonlinearity is obvious near the resonance peak. Besides, in the region near the first-order resonance peak, the energy consumption of nonlinear damping is greater than that of linear damping. The larger the resonance peak is, the more obvious this phenomenon is. However, in the high-frequency domain, the energy consumption of nonlinear damping is almost the same as linear damping. It can be concluded that the resonance peak of the damper can be suppressed by increasing the nonlinear damping at the resonance peak without changing the isolation performance in the high-frequency domain. By choosing reasonable damping orifice 
parameters and fluid viscosities, suppression performance of resonance peak will be better. Besides, we have found that the energy consumption of nonlinear damping at the resonance peak is better than that of linear damping, but this advantage is not very obvious. In the future, through further research, the design parameters of the damper can be optimized, and the nonlinear stiffness and damping can be reasonably selected to achieve more ideal suppression performance at the resonance peak.

\section{Acknowledgements}

This work was supported by the Provincial Joint Fund of Guangdong Province (No.2020A1515110582), Natural Science Foundation of Guangdong Province (No. 2021A1515012051) and National Natural Science Funds for Excellent Yong Scholars of China (No.62022013).

\section{Conflict of interest}

The authors declare that they have no conflict of interest.

\section{Data availability}

Derived data supporting the findings of this study are available from the corresponding author upon reasonable request.

\section{Reference}

[1] Hur, Gyunchul. "Isolation of micro-vibrations due to reaction wheel assembly using a source-pathreceiver approach for quantitative requirements." Journal of Vibration and Control 25.8 (2019): 1424-1435.

[2] Stabile, Alessandro. Electromagnetic Shunt Damper for Spacecraft Micro-Vibration Mitigation. University of Surrey (United Kingdom), 2018.

[3] Davis, Porter, Dave Cunningham, and John Harrell. "Advanced 1.5 Hz passive viscous isolation system." 35th Structures, Structural Dynamics, and Materials Conference. 1994.

[4] Meng, Guang, et al. "Research on micro-vibration control and testing of FY-4 meteorological satellite." SCIENTIA SINICA Physica, Mechanica \& Astronomica 49.2 (2018): 024508. 
[5] Qin, Chao, et al. "Design and optimization of the micro-vibration isolation system for large space telescope." Journal of Sound and Vibration 482 (2020): 115461.

[6] Lee, Dae-Oen, Geeyong Park, and Jae-Hung Han. "Experimental study on on-orbit and launch environment vibration isolation performance of a vibration damper using bellows and viscous fluid." Aerospace Science and Technology 45 (2015): 1-9.

[7] Lee, Dae-Oen, Geeyong Park, and Jae-Hung Han. "Hybrid isolation of micro-vibrations induced by reaction wheels." Journal of Sound and Vibration 363 (2016): 1-17.

[8] Wang, C. X., et al. "Design and experiment of a three-parameter isolation system with optimal damping." Jixie Gongcheng Xuebao/Journal of Mechanical Engineering 51.15 (2015): 90-96.

[9] Wang, Jie, et al. "The interior working mechanism and temperature characteristics of a fluid based micro-vibration damper." Journal of sound and vibration 360 (2016): 1-16.

[10] Shi, Wen-ku, et al. "Modeling and dynamic properties of a four-parameter Zener model vibration damper." Shock and Vibration 2016 (2016).

[11] Wang, Jie, Shougen Zhao, and Dafang Wu. "A numerical study on the performance of nonlinear models of a micro-vibration damper." Shock and Vibration 2014 (2014).

[12] Huang, Xiuchang, et al. "Analytical stiffness model of a fluid-filled U-shaped bellows based threeparameter fluid damper for micro-vibration suppression." Aerospace Science and Technology 69 (2017): 357-369.

[13] Chen, Yanhao, et al. "Dynamics of a passive micro-vibration damper based on a pretensioned plane cable net structure and fluid damper." Journal of Physics: Conference Series. Vol. 744. No. 1. IOP Publishing, 2016.

[14] Likun, Liu, Zheng Gangtie, and Huang Wenhu. "Study of liquid viscosity dampers in octo-strut platform for whole-spacecraft vibration isolation." Acta Astronautica 58.10 (2006): 515-522.

[15] Jiao, Xiaolei, Yang Zhao, and Wenlai Ma. "Nonlinear dynamic characteristics of a micro-vibration fluid viscous damper." Nonlinear Dynamics 92.3 (2018): 1167-1184.

[16] Jiao, Xiaolei, et al. "Research on nonlinear stiffness and damping of bellows-type fluid viscous damper." Nonlinear Dynamics 103.1 (2021): 215-237. 
[17] Dennehy, Cornelius, and Oscar S. Alvarez-Salazar. "Spacecraft micro-vibration: a survey of problems, experiences, potential solutions, and some lessons learned." (2018).

[18] Expansion Joint Manufacturers Association, Inc., "Standards of the Expansion Joint Manufacturers Association, Ninth Edition", 2008.

[19] Langhaar, Henry L. "Steady flow in the transition length of a straight tube." (1942): A55-A58. 